\title{
Metastasis-associated PRL-3 induces EGFR activation and addiction in cancer cells
}

\author{
Abdul Qader Omer Al-aidaroos, ${ }^{1}$ Hiu Fung Yuen, ${ }^{1}$ Ke Guo, ${ }^{1}$ Shu Dong Zhang, ${ }^{2}$ \\ Tae-Hoon Chung, ${ }^{3}$ Wee Joo Chng, ${ }^{3,4}$ and Qi Zeng ${ }^{1,5}$
}

\begin{abstract}
1Institute of Molecular and Cell Biology, Agency for Science, Technology, and Research (A*STAR), Singapore. ${ }^{2}$ Center for Cancer Research and Cell Biology, Queen's University of Belfast, Belfast, United Kingdom. ${ }^{3}$ Haematological Malignancy Genomics Lab, Cancer Science Institute of Singapore, National University of Singapore, Singapore. ${ }^{4}$ Department of Haematology-Oncology, National University Cancer Institute Singapore National University Health System, Singapore. 5Department of Biochemistry, Yong Loo Lin School of Medicine, National University of Singapore, Singapore.
\end{abstract}

\begin{abstract}
Metastasis-associated phosphatase of regenerating liver-3 (PRL-3) has pleiotropic effects in driving cancer progression, yet the signaling mechanisms of PRL-3 are still not fully understood. Here, we provide evidence for PRL-3-induced hyperactivation of EGFR and its downstream signaling cascades in multiple human cancer cell lines. Mechanistically, PRL-3-induced activation of EGFR was attributed primarily to transcriptional downregulation of protein tyrosine phosphatase 1B (PTP1B), an inhibitory phosphatase for EGFR. Functionally, PRL-3-induced hyperactivation of EGFR correlated with increased cell growth, promigratory characteristics, and tumorigenicity. Moreover, PRL-3 induced cellular addiction to EGFR signaling, as evidenced by the pronounced reversion of these oncogenic attributes upon EGFR-specific inhibition. Of clinical significance, we verified elevated PRL-3 expression as a predictive marker for favorable therapeutic response in a heterogeneous colorectal cancer (CRC) patient cohort treated with the clinically approved anti-EGFR antibody cetuximab. The identification of PRL-3-driven EGFR hyperactivation and consequential addiction to EGFR signaling opens new avenues for inhibiting PRL-3-driven cancer progression. We propose that elevated PRL-3 expression is an important clinical predictive biomarker for favorable anti-EGFR cancer therapy.
\end{abstract}

\section{Introduction}

Reversible tyrosine phosphorylation is governed by the balanced action of protein tyrosine kinases and protein tyrosine phosphatases (PTPs). Dysregulation of PTP activity results in aberrant tyrosine phosphorylation, which is frequently implicated in the progression of various diseases including diabetes, rheumatoid arthritis, and cancer (1). Over the past decade, mounting evidence implicates the phosphatase of regenerating liver (PRL) family of PTPs in the metastatic progression of multiple human cancers. The first PRL associated with cancer metastasis was PRL-3 (PTP4A3), which was found to be consistently expressed at high levels in all 18 human colorectal cancer (CRC) liver metastases examined, but at lower levels in the corresponding primary tumors and normal epithelium (2). In a recent study analyzing global gene expression patterns, PRL-3 was again identified as the most significant predictor of liver metastatic recurrence in uveal melanoma patients (3). These reports suggest a key role for PRL-3 in cancer metastasis. To date, elevated PRL-3 expression has been correlated to the metastatic potential and poor prognosis of multiple cancer types, including colorectal, gastric, breast, ovarian, and lung cancers (4). Functionally, PRL-3 promotes multiple stages of malignant transformation including cellular proliferation, invasion, motility, angiogenesis, and survival (5).

PRL-3 has been shown to increase the activity of the PI3K/AKT, MAPK/ERK, and/or SRC pathways in distinct cellular systems (5). Previously, PRL-3 was shown to promote the activation of AKT in DLD-1 colorectal carcinoma cells, with a concomitant downregulation in protein expression levels of the main negative regulator of AKT activity, the phosphatase and tensin homolog

Conflict of interest: The authors have declared that no conflict of interest exists. Citation for this article: J Clin Invest. 2013;123(8):3459-3471. doi:10.1172/JCI66824.
(PTEN) phosphatase (6). However, our recent report (7) suggests that PRL-3 could also increase AKT phosphorylation in cells with PTEN loss-of-function mutations, including A2780 ovarian carcinoma cells (8), implying that PRL-3 might also function independently of PTEN. Among the best-characterized activators of PI3K/AKT signaling are the receptor tyrosine kinases (RTKs). EGFR/ERBB1 is the first of 4 members (ERBB1-4) in the ERBB RTK family. Binding of EGF or its related ligands to the extracellular ligand-binding domain of the ERBB family of receptors leads to the formation of active homo- or heterodimers, which autophosphorylate each other (9). These then serve as hubs for the recruitment and simultaneous activation of various signaling cascades, including the AKT and MAPK pathways, which play critical roles in cell proliferation, survival, adhesion, and migration (10). Consequentially, ERBB ligands and receptors, particularly EGFR and HER2, are frequently overexpressed and/or mutated in many solid tumors, correlating with an unfavorable prognosis, decreased survival, and altered response to chemotherapy (11). Interestingly, effective targeted therapies against many RTKs, including EGFR and HER2/NEU, invariably result in the downregulation of PI3K/AKT signaling (12, 13). Furthermore, in KRAS mutant CRC cells such as DLD-1 and HCT116, RTKs have been demonstrated to exert dominant control over PI3K/AKT signaling (14).

In light of these findings, we hypothesized that PRL-3 might activate RTKs as a proxy in activating multiple oncogenic effectors, including AKT and MAPK, to drive cancer progression. Herein, we describe the PRL-3-induced hyperactivation of the EGFR and its downstream signaling effectors. The addiction of PRL-3-overexpressing cells and tumors to hyperactive EGFR signaling was demonstrated by the hypersensitivity of their growth to EGFR inhibition. Our results reveal a close relationship between 
elevated PRL-3 expression and favorable response to EGFR inhibition, a key finding that could be of immediate clinical relevance in the stratification of patients who are likely to benefit from EGFR-targeted therapies.

\section{Results}

$P R L-3$ overexpression results in byperactivation of EGFR and its downstream signaling pathways. To examine whether PRL-3 overexpression could affect RTK activity, we first engineered A431 epidermoid carcinoma cells to stably express either EGFP (A431-vec) or EGFP-tagged PRL-3 (A431-PRL-3). A431 cells were chosen because they overexpress EGFR and represent a well-established model system routinely used in RTK activation and network modeling studies (15-18). When comparing serum-starved A431-vec and A431-PRL-3 cells, we noted a pronounced increase in tyrosine phosphorylation of multiple protein bands upon PRL-3 overexpression (Figure 1A, lanes 1 and 3). Of these, the most dramatic increase in tyrosyl phosphorylation observed was for a $165-\mathrm{kDa}$ protein that could be further enhanced upon EGF stimulation (Figure 1A, lanes 2 and 4, arrow). Next, using a "phospho-activated" antibody array against 71 unique protein tyrosine kinases (PTKs) (antibody map provided in Supplemental Figure 1; supplemental material available online with this article; doi:10.1172/JCI66824DS1), notable differences in basal activation status between A431-vec control cells (Figure 1B, left panel) and A431-PRL-3 cells (Figure 1B, right panel) were observed. Compared with A431-vec control cells, in A431PRL-3 cells, AXL, EGFR, and ERBB2 RTKs (spot IDs 2E/F, 3E/F, and $5 \mathrm{I} / \mathrm{J}$ in Figure 1, B and C) had the greatest increase in tyrosyl phosphorylation, whereas LYN, TIE2, and ZAP70 (spot IDs 9C/D, $12 \mathrm{C} / \mathrm{D}$, and 13I/J in Figure 1, B and C) had the greatest decrease in tyrosyl phosphorylation.

Since EGFR has an approximate molecular weight of $165 \mathrm{kDa}$ and is known to be overexpressed in A431 cells, we further examined EGFR by Western blotting and found that there was increased activation-associated EGFR tyrosyl phosphorylation (Y1068) in A431-PRL-3 cells (Figure 1D), supporting our phospho-activation antibody array results. Phosphorylation of EGFR was consistently upregulated in A431-PRL-3 cells compared with A431-vec cells regardless of serum supplementation (data not shown). To investigate whether the increased EGFR phosphorylation correlated with enhanced downstream EGFR signaling in A431-PRL-3 cells, EGFR was immunopurified from A431-vec and A431-PRL-3 cells to check for binding of GRB2, an adaptor protein that couples EGFR activation to RAS signaling by direct interaction with phosphorylated Y1068 of the EGFR (19). In non-EGF-stimulated conditions, more GRB2 was associated with EGFR in A431-PRL-3 compared with control A431-vec cells (Figure 1E, lanes 1 and 3). GRB2-associated binding protein 1 (GAB1) is a docking protein which, upon RTK-induced tyrosyl phosphorylation, recruits PI3K to RTKs to activate PI3K/AKT signaling (20). In A431-PRL-3 cells, GAB1 was more highly phosphorylated, with a corresponding increase in AKT phosphorylation (Figure 1F). In support of a functional increase in EGFR activity, phospho-specific antibodies against different activated EGFR downstream targets, including SRC homology 2 domain containing transforming protein 1 (SHC), signal transducer and activator of transcription 1 (STAT1), E twenty-six ETS-like transcription factor 1 (ELK1), and the MAP kinases ERK1/2, JNK, and p38 MAPK, all showed increased phosphorylation in A431-PRL-3 cells compared with A431-vec cells (Figure $1 \mathrm{~F}$ ). These results collectively suggest that PRL-3 increased basal EGFR phosphorylation, activation, and downstream signaling along several signaling pathways, including the MAPK, PI3K/ AKT, and STAT pathways.

PRL-3-mediated hyperactivation of EGFR is partially dependent on SRC activity and may not involve changes in autocrine EGFR ligand secretion. We next investigated the molecular mechanisms to determine how PRL-3 induces EGFR activation. RTK phosphorylation can be regulated by (a) transactivation by other kinases, (b) ligand binding and subsequent autoactivation, and (c) inactivation by intracellular RTK phosphatases. PRL-3 has previously been shown to activate SRC (21), a pleiotropic kinase that is known to modulate the activity and signaling of multiple RTKs, including EGFR, by direct phosphorylation (22). Specifically, SRC has been shown to phosphorylate Y845 of the EGFR to regulate EGFR activity (23). In A431 cells, PRL-3 overexpression resulted in an increase in phosphorylation of both EGFR Y1068 and Y845. Notably, PRL-3induced hyperphosphorylation of EGFR Y845 correlated with a decrease in inhibitory SRC Y530 phosphorylation (Figure 2A, lanes 1 and 2, and Figure 2B). To further investigate the involvement of SRC in EGFR activation, we treated these cells with PP2, a well-characterized inhibitor of SRC family kinases. PP2 treatment resulted in an increase in inhibitory SRC Y530 phosphorylation in both A431-vec and A431-PRL-3 cells. Importantly, SRC inhibition corresponded with a dramatic reduction in both EGFR Y1068 and Y845 phosphorylation in both cell lines. However, it did not abolish the EGFR hyperphosphorylation observed in A431-PRL-3 cells relative to A431-vec cells (Figure 2A, lanes 3 and 4, and Figure 2B). Based on these observations, we reasoned that SRC activity, though critical for sustaining EGFR phosphorylation in these cells, was not the major mechanism for EGFR hyperactivation by PRL-3. In contrast, treatment with AG-1478, a highly specific EGFR kinase inhibitor (24), effectively suppressed EGFR Y1068 and Y845 phosphorylation in both cell lines (Figure 2A, lanes 5 and 6, and Figure 2B). We next examined whether HER2, a classic proto-oncogenic member of the ERBB family, might be involved in EGFR transactivation in PRL-3-overexpressing cells. We detected a dramatic increase in HER2 Y1221/1222 phosphorylation in A431PRL-3 cells relative to A431-vec control cells (Figure 2A, lanes 1 and 2 , and Figure 2B), but this was potently inhibited upon PP2 or AG-1478 treatment in both cell lines (Figure 2A, lanes 3-6, and Figure 2B). A similar observation was made for STAT5 (Figure $2 \mathrm{~A}$, lanes $1-6$, and Figure $2 \mathrm{~B}$ ), a previously characterized SRC substrate (25). These results suggest that HER2, like STAT5, lies downstream of both SRC and EGFR kinase activities and is not responsible for EGFR transactivation by PRL-3.

To investigate the involvement of autocrine factors in regulating EGFR activation in A431-PRL-3 cells, we harvested conditioned culture media from A431-vec and A431-PRL-3 cells to compare differences in secreted autocrine factors. Using an antibody array (antibody map provided in Supplemental Figure 2), no differences were observed in the secretion of 41 unique growth factors between these 2 stable cell pools (Figure 2C), including the EGFR ligands amphiregulin, EGF, heparin-binding EGF-like growth factor (HB-EGF), and TGF- $\alpha$ (9). Collectively, these results indicate that the primary mechanism of PRL-3-specific EGFR hyperactivation lies upstream of SRC activity and may not involve changes in autocrine ligand secretion, at least for the EGFR ligands analyzed.

$P R L-3$ transcriptionally downregulates PTP1B, an EGFR phosphatase, to promote EGFR byperphosphorylation. EGFR dephosphorylation is a physiologically important means of terminating recep- 

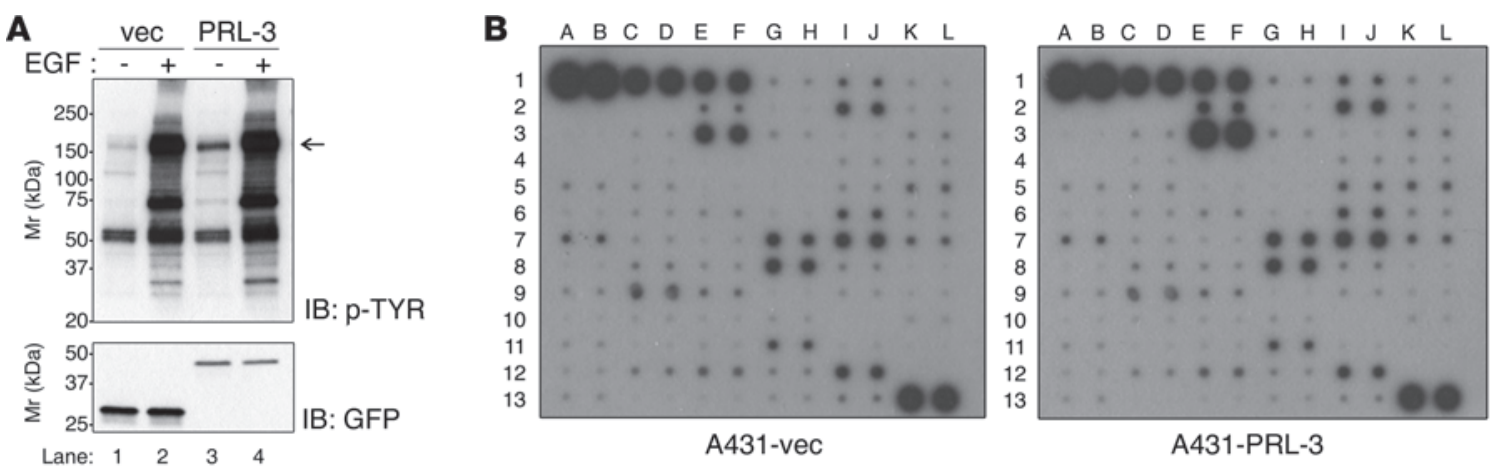

Spot IDs

$2 \mathrm{E}, 2 \mathrm{~F}=\mathrm{AXL}$

$3 E, 3 F=E G F R$

$5 \mathrm{I}, 5 \mathrm{~J}=\mathrm{ERB} 2$

$9 \mathrm{C}, 9 \mathrm{D}=\mathrm{LYN}$

$12 \mathrm{C}, 12 \mathrm{D}=\mathrm{TIE} 2$

$13 \mathrm{I}, 13 \mathrm{~J}=\mathrm{ZAP} 70$

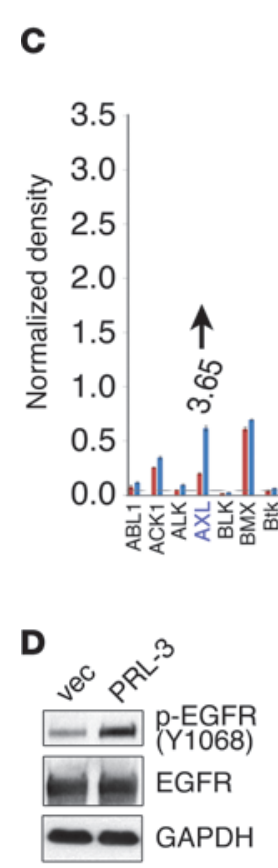

$\stackrel{\uparrow}{\stackrel{\vartheta}{\rho}}$

- A431-vec

- A431-PRL-3

$\uparrow$ Upregulated by PRL-3

$\downarrow$ Downregulated by PRL-3

E

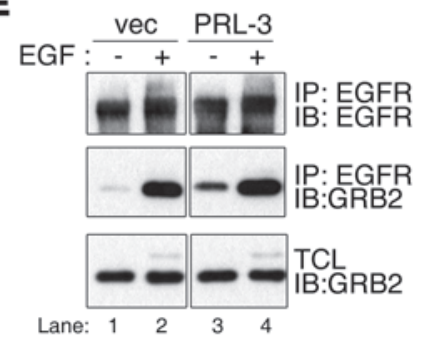

$\mathbf{F}$
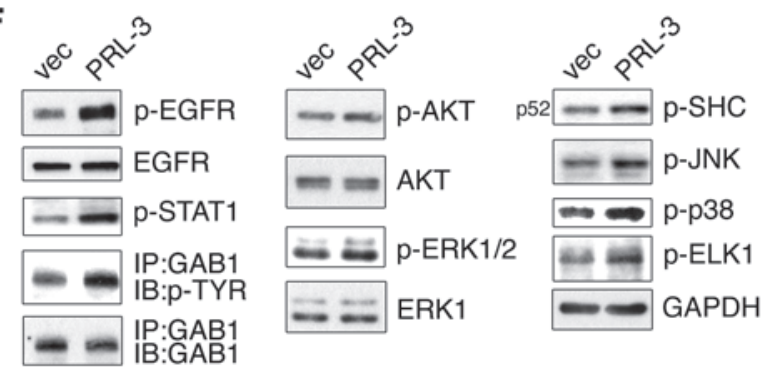

Figure 1

PRL-3 overexpression activates the EGFR in A431 cells. (A) Representative immunoblots of total lysates from serum-starved A431-vec and A431PRL-3 cells with or without EGF (100 ng/ml; 20 minutes) stimulation. The anti-p-Tyr blot was stripped and reprobed with an anti-GFP antibody. Arrow identifies a PRL-3-induced, EGF-responsive tyrosine phosphorylated 165-kDa protein. (B) Total protein lysates from A431-vec (left panel) or A431-PRL-3 (right panel) cells were analyzed using an antibody array against 71 unique PTKs (array map provided in Supplemental Figure 1). The top 3 "PRL-3-activated" or "PRL-3-inactivated" PTKs are indicated on the right, with spot IDs and gene names. (C) Arrays in B were analyzed using densitometry, and fold changes in individual spots were calculated after normalizing to the positive controls on each membrane (mean \pm standard deviation, $n=2$ ). Gene names and fold changes (PRL-3 versus vec) of the top 3 "PRL-3-activated" or "PRL-3-inactivated" PTKs are highlighted. (D) A431-vec or A431-PRL-3 lysates were immunoblotted with antibodies against phosphorylated EGFR (Y1068) and reprobed for total EGFR. GAPDH was used as a loading control. (E) After treatment as in A, lysates from A431-vec and A431-PRL-3 cells were immunoprecipitated (IP) with an anti-EGFR antibody and immunoblotted (IB) with anti-EGFR and anti-GRB2 antibodies. Lane pairs were run on the same gel but were noncontiguous. TCL, total cell lysate. (F) Various EGFR downstream signaling components were analyzed in A431-vec and A431-PRL-3 cells. GAB1 was immunoprecipitated and analyzed using a phosphotyrosine antibody. Blots were probed with the various antibodies as indicated. GAPDH was used as a loading control.

tor signaling, and inhibition of EGFR phosphatases results in EGFR phospho-activation (26). We next investigated the role of PTP1B, a well-characterized tyrosyl-directed EGFR phosphatase $(27,28)$, in PRL-3-induced EGFR hyperactivation. Immunoblot analysis revealed a decrease in $\mathrm{PTP} 1 \mathrm{~B}$ protein expression in A431-PRL-3 cells compared with A431-vec cells, concomitant with an increase in phosphorylated EGFR (Figure 3A). Using quantitative real-time PCR, we found a significant decrease in PTP1B mRNA levels in A431-PRL-3 cells compared with A431vec cells. Importantly, the extent of reduction in PTP1B mRNA levels correlated well with a decrease in PTP1B protein expression (Figure 3B). This result suggests that PRL-3 might be reg- 
A

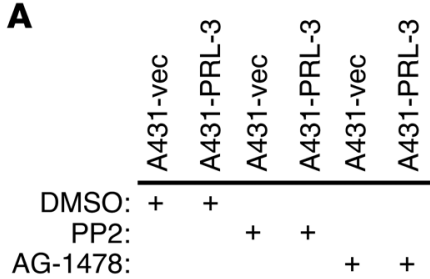

AG-1478:

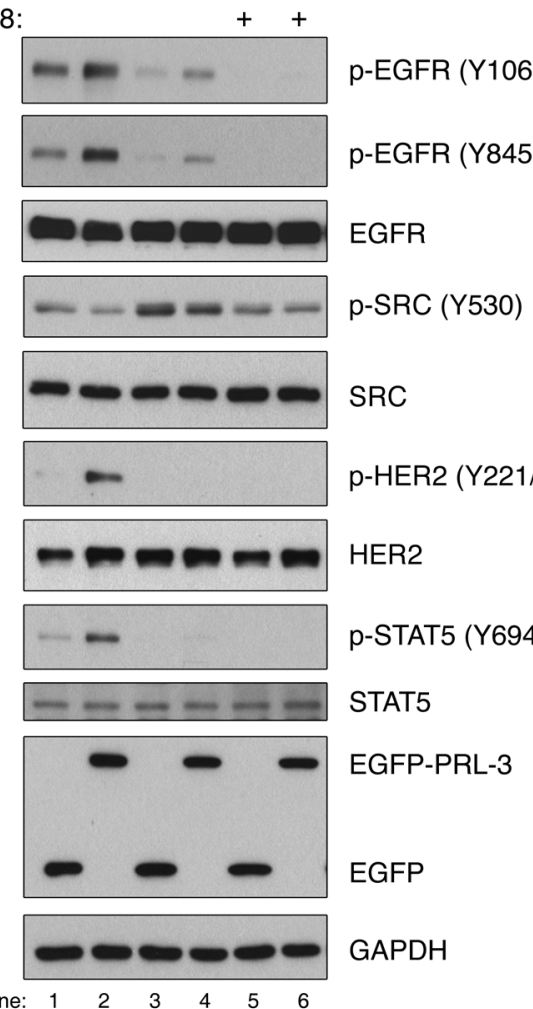

B
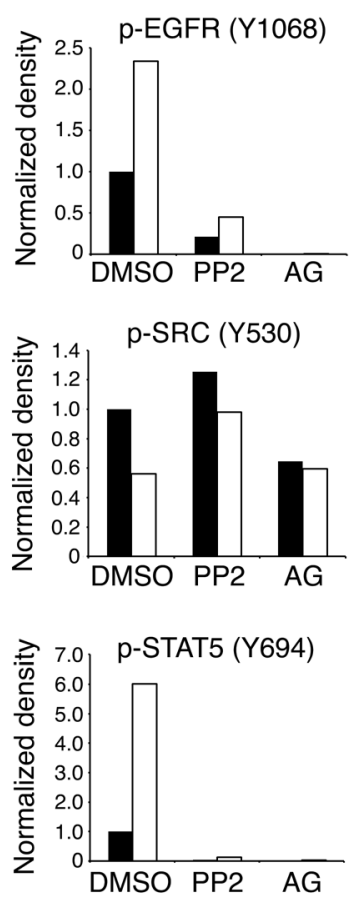

C

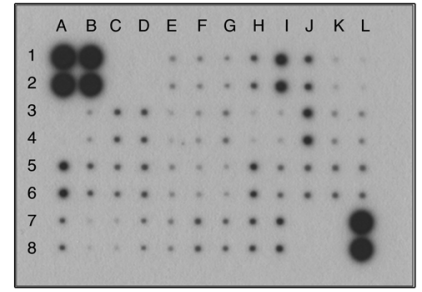

A431-vec
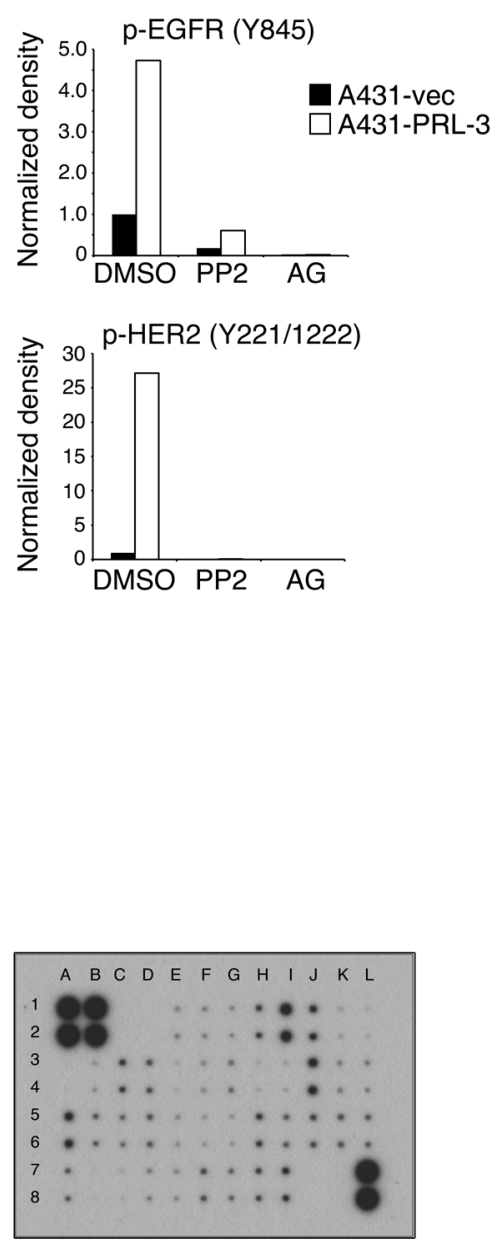

A431-PRL-3

Figure 2

PRL-3-induced EGFR activation is partially dependent on SRC activity and may not involve changes in autocrine stimulation. (A) Representative immunoblots from A431-vec and A431-PRL-3 cells treated with DMSO (16 hours), PP2 (16 hours), or AG-1478 (75 minutes) before lysates were collected and analyzed. Phosphoprotein blots were stripped and reprobed for their total protein counterparts. GAPDH was used as a loading control. (B) Quantitation of selected immunoblot densities in A. Phosphoprotein band densities were normalized to their total protein counterparts. Black bars represent A431-vec; white bars represent A431-PRL-3. (C) Serum-free conditioned media (20 hours) from A431-vec and A431-PRL-3 cells were collected and analyzed using an antibody array against 41 different growth factors (array map provided in Supplemental Figure 2). No significant differences were quantitated using densitometry (data not shown).

ulating PTP1B expression at the transcript level. To examine whether PRL-3-driven downregulation of PTP1B was a general phenomenon, this finding was validated in 3 other human cancer cell lines of different tissue origins and with differential EGFR protein expression levels: MDA-MB-468 breast cancer cells (high EGFR levels), HCT116 colon cancer cells (moderate EGFR levels), and A2780 ovarian cancer cells (low EGFR levels) (Supplemental Figure 3A). Pooled colonies of MDA-MB-468 breast cancer cells overexpressing EGFP-PRL-3 (MDA-MB-468PRL-3) revealed higher EGFR phosphorylation and reduced PTP1B protein expression compared with control cells (MDAMB-468-vec) (Figure 3C). Again, the expression of PTP1B mRNA and protein was downregulated by similar degrees in these cells (Figure 3D). To further validate the influence of PRL-3 on EGFR activation, we analyzed the EGFR activation status in HCT116 cells, which abundantly express endogenous PRL-3, upon stable expression of either a scrambled (shSCR) or a PRL-3-specific shRNA (shPRL-3) construct. EGFR phosphorylation was clearly reduced upon knockdown of endogenous PRL-3, correlating with an upregulation of PTP1B expression levels (Figure 3E). Consistently, we also observed a decrease in PTP1B expression in A2780 cells upon PRL-3 overexpression (Supplemental Figure 3B). However, phosphorylated EGFR could not be detected in our A2780 immunoblots, likely due to the low EGFR expression in this cell line (Supplemental Figure 3A). Collectively, these results suggest a close relationship between the suppression of PTP1B expression and EGFR hyperphosphorylation in response to PRL-3 overexpression.

To verify whether the downregulation of PTP1B was caused by PRL-3 overexpression and not by a negative feedback loop due to EGFR hyperactivation, we treated MDA-MB-468-vec and MDAMB-468-PRL-3 cells with 3 distinct EGFR inhibitors: AG-1478, 
A

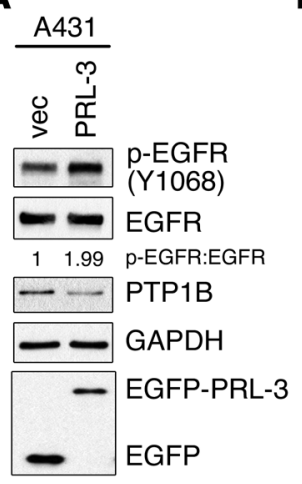

$\mathbf{F}$

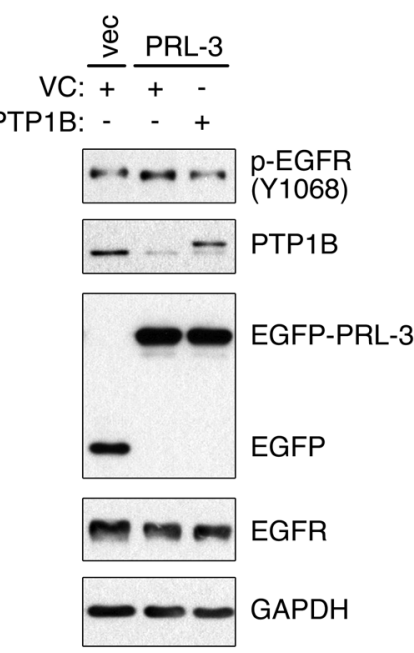

B

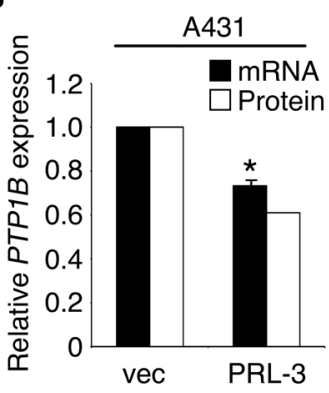

C MDA-MB-

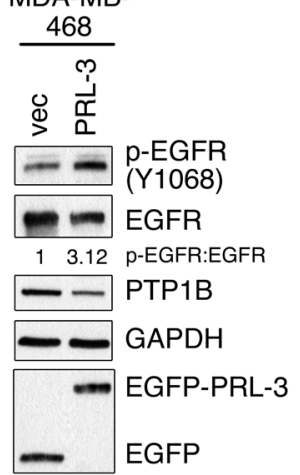

D

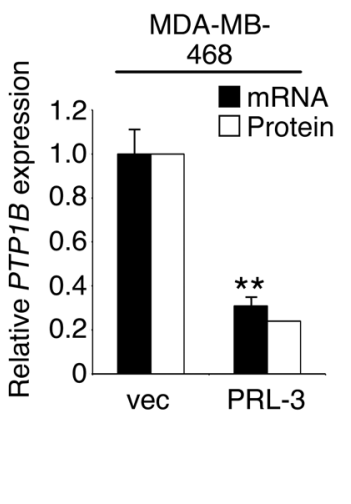

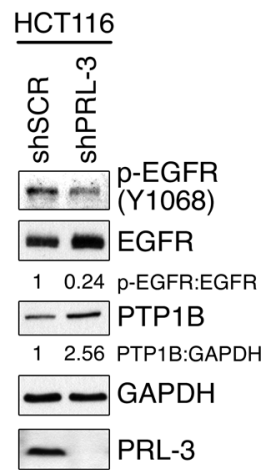

G

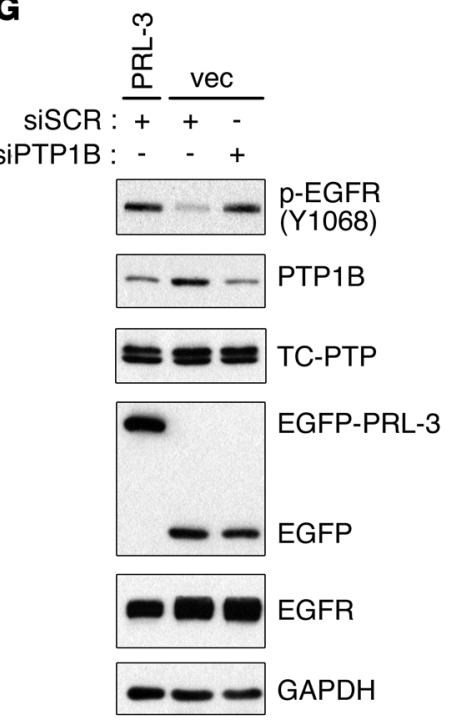

H

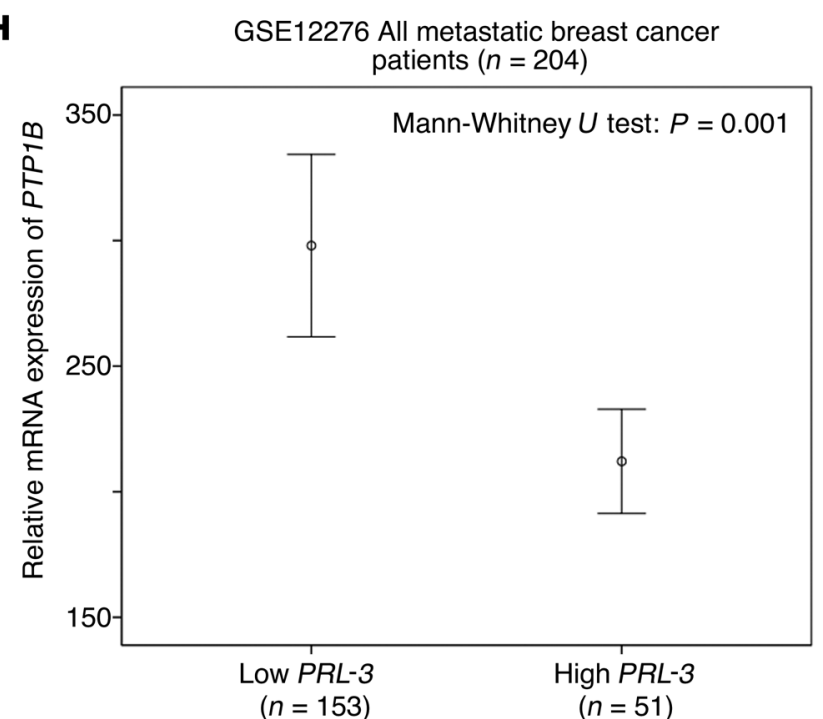

mRNA expression levels of $P R L-3$

\section{Figure 3}

PRL-3 induces EGFR hyperphosphorylation via PTP1B downregulation. (A) A431-vec and A431-PRL-3 cells were analyzed for PTP1B expression and EGFR (Y1068) phosphorylation. Numbers below EGFR immunoblots reflect the ratio of p-EGFR/EGFR blot densities. GAPDH was used as a loading control. (B) Comparison of relative PTP1B mRNA expression (biological triplicate; mean \pm standard deviation; paired Student's $t$ test, ${ }^{*} P=0.012$ ) versus protein expression (densitometric quantitation of PTP1B bands in A) in A431-vec and A431-PRL-3 cells. GAPDH mRNA expression and protein expression, respectively, were used for normalization. (C) MDA-MB-468-vec and MDA-MB-468-PRL-3 cells were analyzed for PTP1B expression and EGFR phosphorylation as in A. (D) Comparison of relative PTP1B mRNA expression (mean \pm standard deviation; Student's $t$ test, ${ }^{* *} P<0.001$ ) versus protein expression in MDA-MB-468-vec and MDA-MB-468-PRL-3 cells, analyzed as in B. (E) HCT116 cells stably expressing scrambled (shSCR) or PRL-3-specific shRNA (shPRL-3) were analyzed for PTP1B expression and EGFR phosphorylation as in A. (F) MDA-MB-468-vec and MDA-MB-468-PRL-3 cells transiently transfected (18 hours) with either empty vector (VC) or FLAG-tagged PTP1B constructs were analyzed for EGFR phosphorylation. (G) MDA-MB-468-vec and MDA-MB-468-PRL-3 cells transiently transfected (72 hours) with control nonspecific siRNA (siSCR) or PTP1B-targeting siRNA (siPTP1B) were analyzed for EGFR phosphorylation. T cell PTP (TC-PTP), the closest homolog of PTP1B, served as a control for siRNA specificity. GAPDH was used as a loading control. (H) Stratified PRL-3 mRNA expression levels were negatively correlated to raw PTP1B mRNA expression levels in metastatic breast cancer patient samples $(n=204$; Mann-Whitney $U$ test, $P=0.001$ ).

erlotinib (29), or cetuximab (30). These inhibitors failed to restore the reduced PTP1B levels in the MDA-MB-468-PRL-3 cells back to those in the MDA-MB-468-vec control cells (Supplemental Figure 3C), suggesting that PRL-3-induced PTP1B downregulation lies upstream of EGFR hyperphosphorylation. Next, to validate whether alterations in PTP1B expression are causally involved in PRL-3-induced EGFR hyperphosphorylation, MDA-MB-468-vec and MDA-MB-468-PRL-3 cells, which demonstrated the largest difference in PTP1B levels upon forced PRL-3 expression (Figure
3C), were transiently transfected with either FLAG-tagged PTP1B or PTP1B-specific siRNA sequences. When we rescued PTP1B expression in MDA-MB-468-PRL-3 cells using transient FLAGPTP1B overexpression, EGFR phosphorylation decreased to the levels observed in MDA-MB-468-vec control cells (Figure 3F). This effect was not specific to PRL-3-overexpressing cells, as PTP1B overexpression in MDA-MB-468-vec control cells also resulted in EGFR dephosphorylation (Supplemental Figure 3D). Conversely, PTP1B depletion in MDA-MB-468-vec cells resulted in an increase 


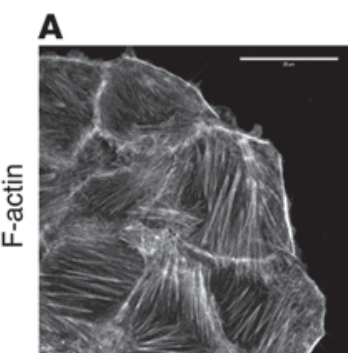

MDA-MB-468-vec

+ DMSO
B

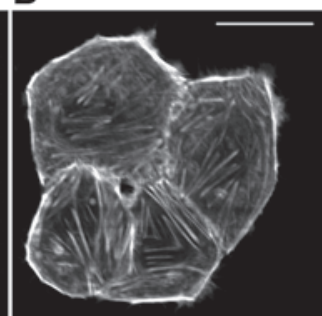

MDA-MB-468-vec

+ erlotinib

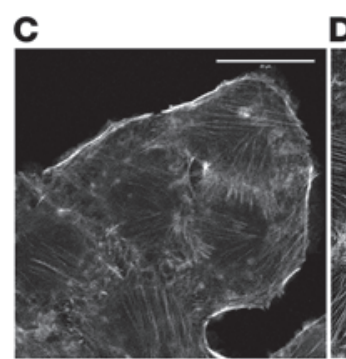

MDA-MB-468-PRL-3 + DMSO

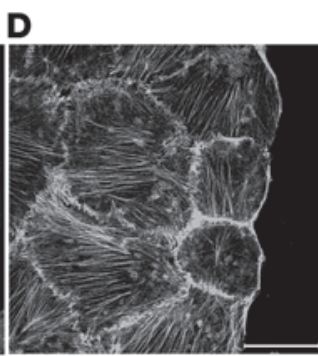

MDA-MB-468-PRL-3

+ erlotinib
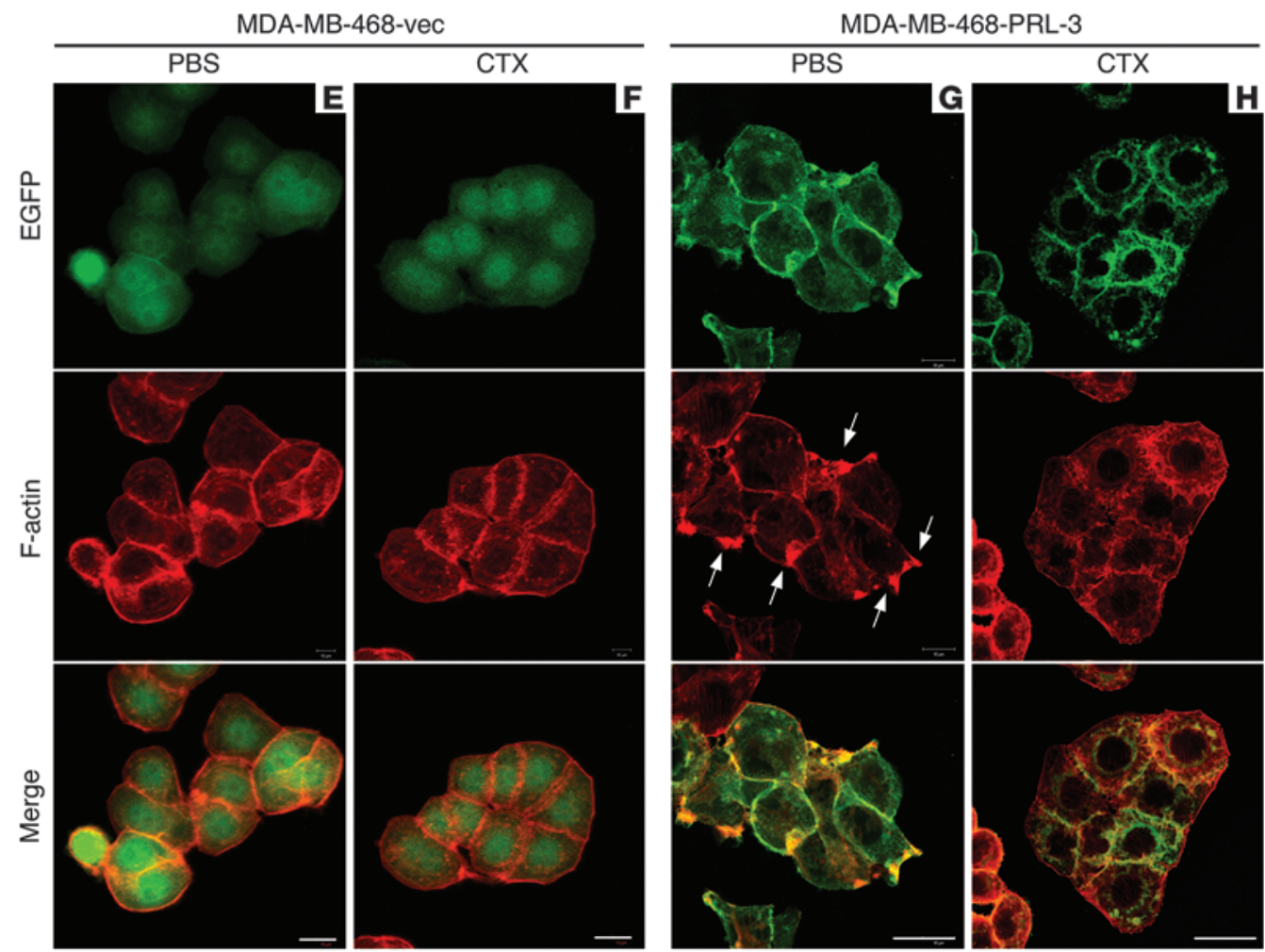

Figure 4

EGFR-specific inhibitors block PRL-3-induced dissolution of stress fibers and F-actin enrichment at membrane protrusions. (A-D) MDA-MB468-vec (A and B) or MDA-MB-468-PRL-3 (C and D) cells were seeded on glass coverslips and incubated with $0.1 \%$ DMSO (A and $\mathbf{C})$ or $2 \mu \mathrm{M}$ erlotinib (B and D) 16 hours prior to fixing. Fixed cells were stained with phalloidin to visualize F-actin stress fibers. Scale bars: $20 \mu \mathrm{m}$. (E-H) MDA-MB-468-vec (E and F) or MDA-MB-468-PRL-3 (G and $\mathbf{H})$ cells were seeded on glass coverslips and incubated with PBS (E and $\mathbf{G})$ or 100 nM cetuximab ( $\mathbf{F}$ and $\mathbf{H}) 16$ hours prior to fixing. Fixed cells were stained with phalloidin to visualize F-actin enrichment. Arrows indicate sites of F-actin-rich membrane projections. Green, EGFP signal; red, F-actin. Scale bars: $20 \mu \mathrm{m}$.

in EGFR phosphorylation to the levels seen in MDA-MB-468PRL-3 cells (Figure 3G). Notably, depletion of PTP1B in MDAMB-468-PRL-3 cells resulted in further enhancement of EGFR hyperphosphorylation (Supplemental Figure 3E), suggestive of a dose dependence of EGFR phosphorylation on PTP1B expression. Although the precise molecular mechanism remains to be defined in future studies, our results here suggest that PRL-3 downregulates PTP1B in order to inhibit EGFR dephosphorylation, thereby resulting in EGFR hyperphosphorylation.

PRL-3 and PTP1B $m R N A$ expression is inversely correlated in a cohort of metastatic breast cancer patients. To investigate the clinical relevance of the relationship between PRL-3 and PTP1B, two previously unrelated phosphatases, we analyzed a publicly available microarray dataset generated from a cohort of 204 metastatic breast cancer patients. In this dataset, HB-EGF was identified as a key mediator of breast cancer metastasis to the brain, implicating EGFR signaling in the metastatic dissemination of primary tumors in these patients (31). Consistent with the prometastatic role of PRL-3, we found that patients with high PRL-3 mRNA expression had significantly shorter disease-free survival times (median survival $=20.5$ months, 95\% CI = 16.0-25.0) compared with those with low PRL-3 mRNA expression in their primary tumors (median survival $=$ 28.1 months, $95 \% \mathrm{CI}=24.4-31.8$ ), with a hazard ratio $(\mathrm{HR})$ of 1.45 $(95 \% \mathrm{CI}=1.05-2.00, P=0.024)$ between them. Importantly, $P R L-3$ and $P T P 1 B$ transcript levels in this dataset were significantly and inversely correlated with each other (Spearman's rank test, 


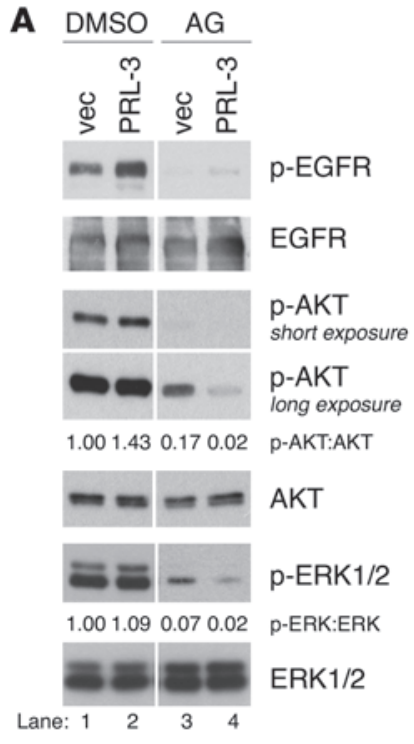

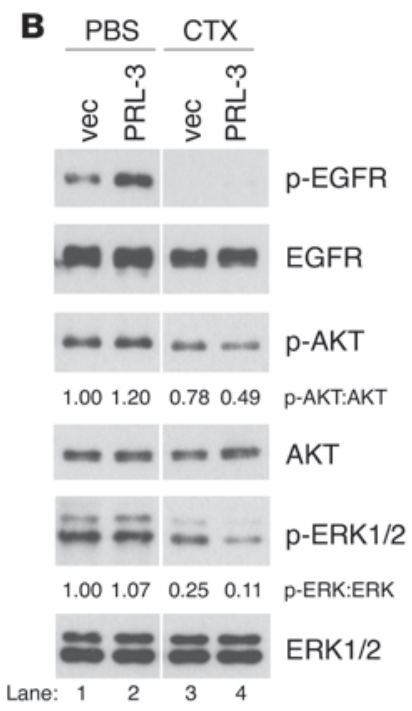
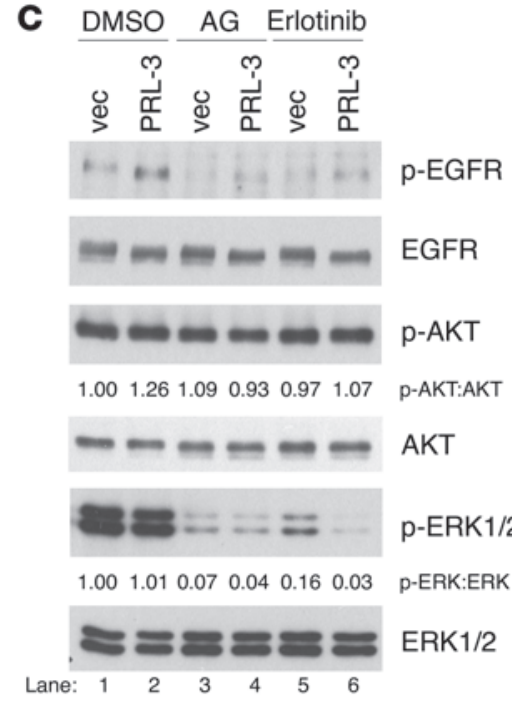
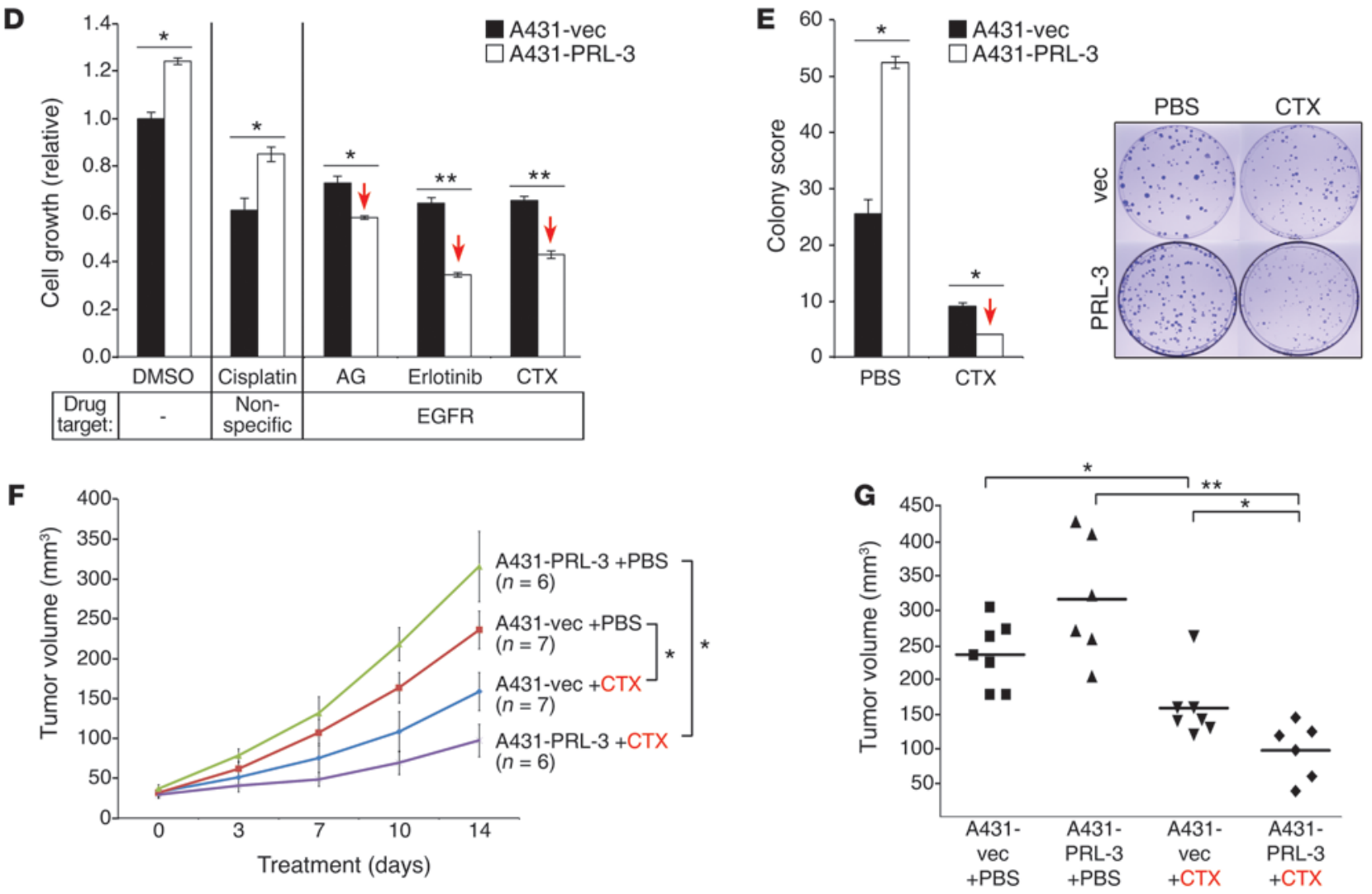

\section{Figure 5}

PRL-3-overexpressing cells are hypersensitive to EGFR-specific inhibitors. (A) Representative immunoblots of serum-starved A431-vec and A431-PRL-3 cells treated with DMSO or AG-1478 (AG) 75 minutes before lysis. Lane pairs were run on the same gel but were noncontiguous. Normalized densitometric ratios of phosphoproteins/total proteins are indicated below selected blots. (B) Representative immunoblots of serum-starved A431-vec and A431-PRL-3 treated with PBS or cetuximab (CTX) 24 hours before lysis. Lane pairs were run on the same gel but were noncontiguous. (C) Representative immunoblots of serum-starved MDA-MB-468-vec and MDA-MB-468-PRL-3 cells treated with DMSO, AG-1478 (75 minutes), or erlotinib (3 hours) before lysis. (D) A431-vec or A431-PRL-3 cell growth after 48-hour exposure to DMSO, cisplatin, AG-1478, erlotinib, or cetuximab in 0.5\% FBS medium (mean \pm standard deviation; $n=3$; unpaired Student's $t$ test, ${ }^{*} P<0.05,{ }^{* *} P<0.001$ ). Black bars, A431-vec; white bars, A431-PRL-3. Arrows indicate a hypersensitive response. (E) A431-vec and A431-PRL-3 cell colony score after 11 days of treatment with PBS or $20 \mathrm{nM}$ cetuximab in $10 \%$ FBS medium (mean \pm standard deviation; $n=2$; unpaired Student's $t$ test, ${ }^{*} P<0.05$ ). Arrow indicates a hypersensitive response. Representative images of colony formation are shown in the right panel. (F) Growth curves of A431-vec or A431-PRL-3 xenograft tumors treated i.p. with 1 mg cetuximab or $200 \mu l$ PBS twice weekly for 2 weeks (mean \pm standard deviation; $\left.{ }^{*} P<0.05\right)$. (G) Comparison of day 14 tumor volumes from $\mathbf{F}\left(\right.$ Student's $t$ test, $\left.{ }^{\star} P<0.05,{ }^{* \star} P<0.001\right)$. 

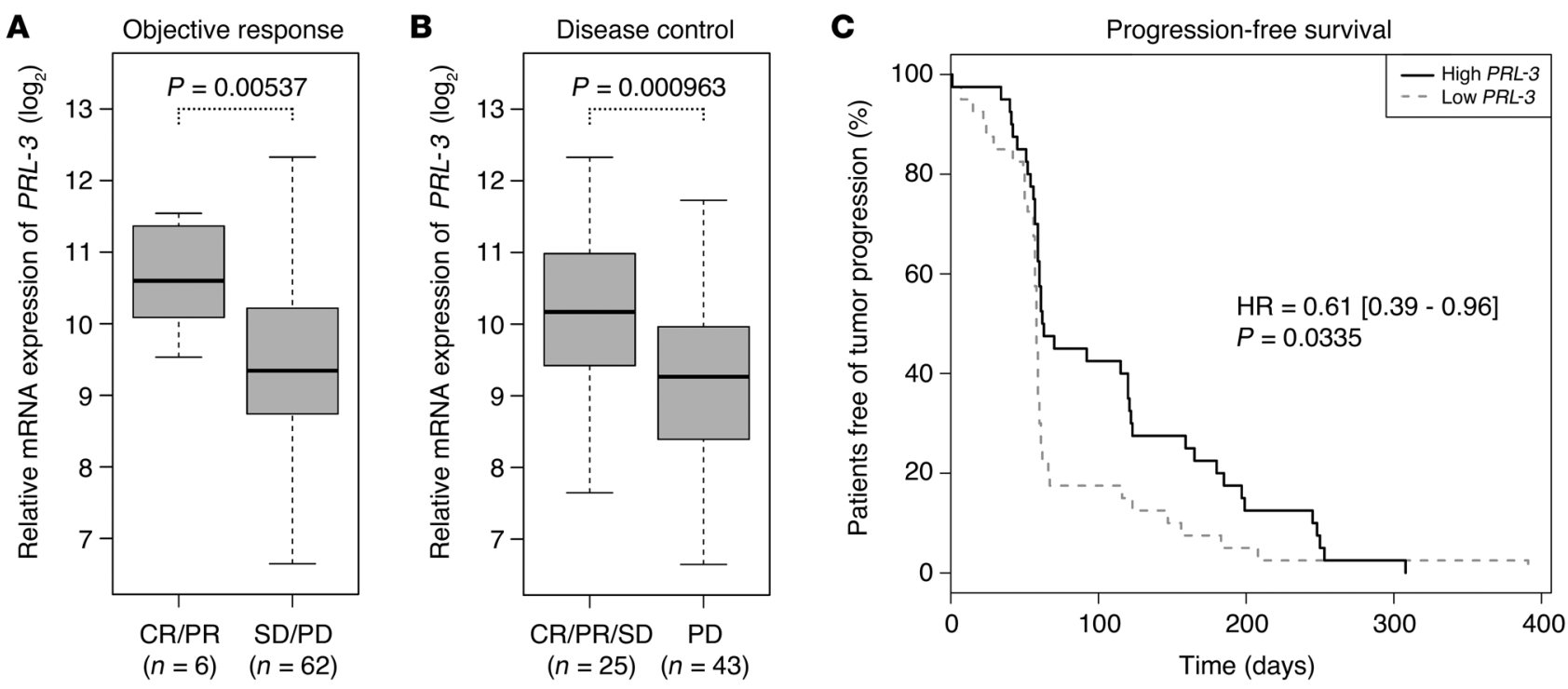

Figure 6

High $P R L-3$ expression levels correlate with a favorable therapeutic response in cetuximab-treated CRC patients. $P R L-3$ mRNA expression levels positively correlate with $(\mathbf{A})$ objective response $(n=68$; Student's $t$ test, $P<0.01)$ and (B) disease control $(n=68$; Student's $t$ test, $P<0.001)$ in cetuximab-treated CRC patients. Note that the relative mRNA expression of $P R L-3$ is presented on a logarithmic $\left(\log _{2}\right)$ scale. (C) High $P R L-3 \mathrm{mRNA}$ expression is associated with longer progression-free survival times in cetuximab-treated CRC patients $(n=68$; Cox regression analysis, $P<0.05)$.

$r=-0.168, P=0.016)$. Indeed, patients expressing high levels of $P R L-3$ had significantly lower $P T P 1 B$ expression (mean \pm SEM $=$ $212.1 \pm 10.3$ ) compared with those expressing low levels of $P R L-3$ (mean $\pm \mathrm{SEM}=298.0 \pm 18.4$; Mann-Whitney $U$ test, $P=0.001$ ) (Figure $3 \mathrm{H}$ ), indicating an antagonistic relationship between $P R L-3$ and $P T P 1 B$ expression in clinical metastatic breast cancer samples and supporting our in vitro observations.

$P R L$-3-induced stress fiber dissolution and F-actin enrichment at sites of membrane protrusions can be blocked by EGFR inbibition. Since the above-described patient dataset consisted of samples from metastatic breast cancer patients, we next investigated the biological consequences of EGFR activation by PRL-3 on the motile phenotype of the metastatic breast cancer cell line MDA-MB-468. Cell migration involves the orchestrated assembly and disassembly of actin filaments (32). Using immunofluorescence analysis, we observed a marked decrease in cytoskeletal F-actin stress fibers in MDA-MB-468-PRL-3 cells (Figure 4C) compared with MDAMB-468-vec control cells (Figure 4A). This observation is in agreement with previously published results (6) and is consistent with increased cell migration (33). To validate whether EGFR activation by PRL-3 could be involved in the decrease of actin stress fibers, we used erlotinib to treat these cells. Erlotinib treatment did not affect stress fiber formation in MDA-MB-468-vec cells (Figure 4B), but did effectively block stress fiber dissolution in MDA-MB-468PRL-3 cells (Figure 4D) and returned these cells to a similar state as seen in the vector control cells (Figure 4A). Besides modulation of stress fiber assembly, EGF stimulation has been previously shown to stimulate cell migration by the formation of actin-rich membrane protrusions at the leading edge of migratory cells in a PI3K-dependent manner (34). Compared with MDA-MB-468-vec cells (Figure 4E), we noted a pronounced increase in F-actin-rich membrane protrusions at the leading edge of MDA-MB-468-PRL-3 cells (Figure 4G). Similarly, PRL-3-induced membrane projections were essentially blocked by EGFR inhibition using cetuximab (Fig- ure $4 \mathrm{H})$. Importantly, whereas there was no observed difference with regard to both stress fiber formation and membrane protrusions in MDA-MB-468-vec cells upon EGFR inhibition (Figure 4, A, B, E, and F), MDA-MB-468-PRL-3 cells responded dramatically to EGFR inhibition (Figure 4, C, D, G, and H). Collectively, these results suggest that PRL-3 enhances stress fiber disassembly and induces leading-edge F-actin-rich membrane protrusions in an EGFR-dependent manner.

$P R L-3-$ overexpressing cells are bypersensitive to EGFR inbibition. Because PRL-3-overexpressing cells displayed enhanced activation of the signaling components involved in prosurvival and proliferative pathways, including AKT and ERK1/2, we investigated whether inhibition of EGFR could potentially abrogate these oncogenic events. Treatment of A431-vec and A431-PRL-3 cells with AG-1478 effectively reduced AKT and ERK1/2 phosphorylation levels in both cell types. Unexpectedly, the reduction in phosphorylated AKT and ERK1/2 upon EGFR kinase inhibition by AG-1478 was much greater in A431-PRL-3 cells than in A431-vec control cells (Figure 5A, lanes 3 and 4). To confirm this phenomenon, we treated A431-vec and A431PRL-3 cells with a different EGFR inhibitor, cetuximab. Treatment with a physiologically relevant cetuximab dose potently inhibited EGFR phosphorylation in both cell lines. Significantly, phosphorylated AKT and ERK1/2 were again reduced by a greater extent in cetuximab-treated A431-PRL-3 cells compared with A431-vec cells (Figure 5B, lanes 3 and 4). To rule out cell-specific effects, we treated MDA-MB-468-vec and MDA-MB-468-PRL-3 cells with the EGFR inhibitors AG-1478 and erlotinib. In agreement with previous reports $(35,36)$, no significant changes in phosphorylated AKT were observed upon EGFR inhibition in these PTEN-deficient MDA-MB-468 cells (Figure 5C, lanes 1-6). However, phosphorylated ERK1/2 was decreased by a greater extent in MDA-MB-468-PRL-3 cells compared with MDA-MB-468-vec cells upon EGFR inhibition 
A

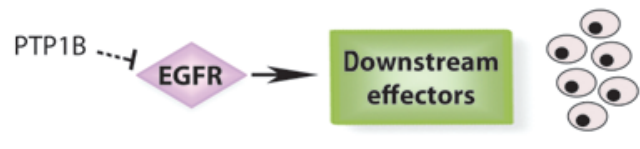

C

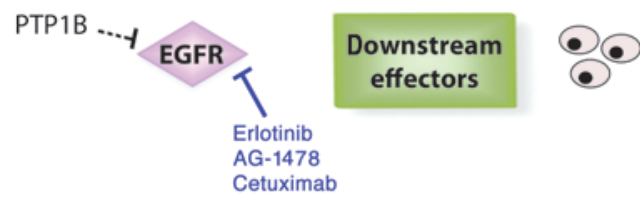

B PRL-3 induces hyperactivation of the EGFR

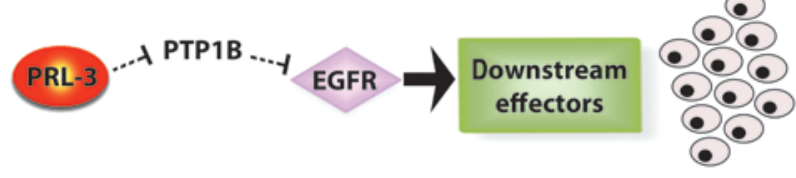

D PRL-3 induces hypersensitivity to EGFR inhibition

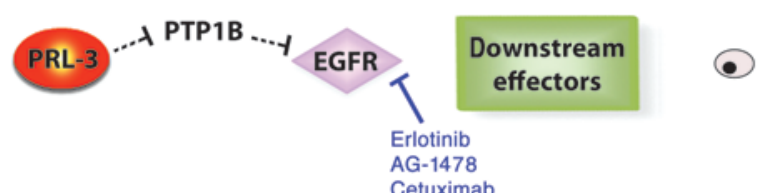

Figure 7

Proposed model of PRL-3-induced oncogenic addiction to hyperactivated EGFR. (A and B) Compared with non-PRL-3-overexpressing cells (A), PRL-3 overexpression (B) induces constitutive hyperactivation of EGFR via the downregulation of PTP1B, promoting the growth of "EGFR-addicted" cancer cells. (C and D) Compared with non-PRL-3-overexpressing cells (C), PRL-3-overexpressing EGFR-addicted cells (D) are hypersensitive to EGFR inhibition.

(Figure 5C, lanes 3-6). Collectively, these results suggest that the hypersensitive response to EGFR inhibition in PRL-3-overexpressing cells is likely a general property.

The hypersensitivity of PRL-3-expressing cells to EGFR inhibition was reminiscent of "oncogene addiction," a phenomenon in which the growth and survival of cancer cells may be overly dependent on one or a few dominant, driver oncogenes (37). We thus hypothesized that the inhibition of EGFR in such PRL-3-driven "EGFR-addicted" cells might be a specific and therapeutically exploitable means to inhibit their growth. We first analyzed cell growth in the presence of the general proliferation inhibitor cisplatin (38) or EGFR-specific inhibitors (AG-1478, erlotinib, and cetuximab). In the absence of inhibitor treatment, A431-PRL-3 cells grew significantly faster than A431-vec cells (Figure 5D). Cisplatin treatment resulted in an even decrease in both A431-vec and A431PRL-3 cell proliferation (Figure 5D), without any PRL-3-specific hypersensitive response to this general proliferation inhibitor. In sharp contrast, all 3 EGFR-specific inhibitors (AG-1478, erlotinib, and cetuximab) led to a significantly greater decrease in A431PRL-3 cell growth compared with A431-vec cells (Figure 5D; red arrows indicate a hypersensitive response). Interestingly, chemical inhibition of SRC, AKT, or ERK1/2 (using PP2, perifosine, or U0126, respectively), despite inhibiting A431-PRL-3 cell proliferation, failed to reproduce such a PRL-3-induced hypersensitive response (Supplemental Figure 4). Next, we used a monolayer clonogenicity assay to investigate the ability of cetuximab to inhibit colony formation of A431-vec and A431-PRL-3 cells. Although A431-PRL-3 cells formed more colonies than A431-vec cells under control (PBS-treated) conditions, this was significantly reversed upon cetuximab treatment (Figure 5E). Finally, to test whether PRL-3 could endow cancer cells with increased anti-EGFR agent susceptibility in vivo, we treated established xenograft tumors of A431-vec or A431-PRL-3 cells in nude mice with PBS or cetuximab twice weekly for 2 weeks and monitored tumor growth. We found that A431-vec tumors grew slower than A431-PRL-3 tumors in PBStreated mice, yet this was markedly reversed in cetuximab-treated mice (Figure 5F). Importantly, an analysis of tumor volumes at the end of the experiment (day 14) revealed significantly smaller A431-PRL-3 tumors compared with A431-vec tumors in the cetuxi- mab-treated mice (Figure 5G). In summary, our results suggest that PRL-3 induces hypersensitivity to EGFR inhibition both in vitro and in vivo due to hyperactive EGFR signaling addiction.

PRL-3 expression is a predictor of a favorable therapeutic response in cetuximab-treated CRC patients. To investigate the clinical relevance of our observations, we tested whether PRL-3 mRNA expression levels correlated with tumor sensitivity to anti-EGFR drug treatment in a microarray dataset from a CRC patient cohort prior to treatment with cetuximab monotherapy (39). Patients' tumors that responded better to cetuximab therapy (complete or partial response) had a significantly higher median expression level of $P R L-3$ compared with those that did not respond to the same treatment $(P<0.01)$ (Figure 6A). Furthermore, a significantly higher median expression level of $P R L-3$ was also observed in patients with controlled disease as compared with patients who had progressive disease $(P<0.001)$ (Figure 6B). Indeed, patients with a high level of PRL-3 expression had an overall response rate (ORR) of $17 \%$ and a disease control rate (DCR) of $53 \%$, both of which were higher than what was observed in patients with a low level of $P R L-3$ expression (ORR $=0 \%$, $P=0.026$; DCR $=19 \%, P=0.005)$. Importantly, high $P R L-3$ expression was associated with a longer progression-free survival rate in cetuximab-treated patients compared with those who had low $P R L-3$ expression $(\mathrm{HR}=0.61,95 \% \mathrm{CI}=0.39-0.96, P=0.034)$ (Figure 6C). Our clinical observations are in agreement with a previous report (40) showing that elevated PRL-3 (PTP4A3) expression ranked first, sixth, and fourth (of 110 genes) in terms of correlation with a favorable objective response, disease control, and progressionfree survival, respectively, in an independent cetuximab-treated CRC patient cohort. Collectively, these clinical results support our in vitro observations that a high level of PRL-3 expression induces sensitivity to EGFR inhibition due to addiction to activated EGFR. Interestingly, analysis of PRL-3 expression levels in a microarray dataset of gefitinib-sensitive or gefitinib-resistant NSCLC cell lines (41) also revealed significantly higher PRL-3 expression levels in gefitinib-sensitive cells (Supplemental Figure 5). Taken together, our results suggest that PRL-3-induced addiction to EGFR might be a general property in multiple cancer types and indicate that PRL-3 expression levels could be a useful predictive biomarker for favorable anti-EGFR therapy response in patients. 


\section{Discussion}

In this study, we present evidence for PRL-3-induced hyperactivation of EGFR and its downstream effectors. We characterized the downregulation of an EGFR phosphatase, PTP1B, as a key mechanism involved in PRL-3-mediated hyperphosphorylation of EGFR. Indeed, an inverse relationship was found between PRL-3 and PTP1B expression in various cell lines as well as in clinical breast cancer samples, thereby establishing a novel relationship between these two phosphatases. Functionally, we found that pharmacological inhibition of EGFR signaling could potently inhibit PRL-3-enhanced cell and tumor growth, revealing a profound hypersensitivity of PRL-3-overexpressing cells and tumors to EGFR inhibition. Clinically, the translational relevance of these findings is that elevated PRL-3 expression could predict a favorable antiEGFR therapeutic response in CRC patients, in corollary suggesting a novel approach to the inhibition of dual PRL-3/EGFR-positive tumors by targeting PRL-3-induced EGFR addiction. Figure 7 summarizes our working model on how PRL-3 causes hyperactivation of oncogenic EGFR signaling, as well as the hypersensitivity of such EGFR-addicted cancer cells to EGFR inhibition.

The aberrant activation of RTKs has been linked to malignant progression of many human cancers (42). Our results suggest that PRL-3 exhibits pleiotropic signaling by activating EGFR, a key initiator of multiple signaling cascades (10). Despite pronounced EGFR activation by PRL-3, we noted that the extent of increase in EGFR phosphorylation did not correlate linearly with that of the various downstream effectors analyzed (Figure $1 F$ ). It could be that our use of pooled cell populations, the concurrent downregulation of several kinase activities (including LYN, TIE2, and ZAP70) (Figure 1, B and C), and/or the existence of negative feedback loops (43) resulted in a "masking" of the linearity between EGFR activation and downstream pathway activity in response to PRL-3 overexpression. Nonetheless, our results herein provide a potential explanation for previous reports of PRL-3-mediated activation of the PI3K/AKT, SRC/STAT, and RAS/MAPK pathways (5), all of which lie downstream of RTK signaling (44).

PTP1B has been reported to reduce the phosphorylation of multiple EGFR signaling pathway components, including PLC$\gamma 1$, GAB1, SHP2, SHP, and EGFR itself (45). Although the involvement of other EGFR phosphatases (26) cannot be discounted, our study suggests that PTP1B is the primary EGFR phosphatase causally involved in PRL-3-driven EGFR hyperphosphorylation. The reduced levels of PTP1B in A431 and MDA-MB-468 cells overexpressing PRL-3 and the accumulation of PTP1B in PRL-3depleted HCT116 cells were, in each case, concomitant with an increase or decrease in EGFR phosphorylation, respectively. Furthermore, changes in PTP1B mRNA expression alone, as supported by overexpression "rescue" experiments and siRNA-mediated depletion (Figure 3, F and G), appear sufficient to phenocopy PRL-3-induced EGFR hyperphosphorylation. PTP1B expression is regulated by several transcription factors, including YB1 (46), HIF (47), and the EGR1 and SP family of transcription factors (48). It is plausible that PRL-3 suppresses PTP1B mRNA expression via the regulation of some of these transcription factors. Alternatively, in light of the recently characterized role of PRL-3 as an epigenetic regulator (49), PRL-3 might induce modifications on the $P T P 1 B$ promoter/enhancer locus to suppress $P T P 1 B$ transcription. Although further studies are required to clarify the precise mechanism of PTP1B mRNA downregulation by PRL-3, our results lend weight to the existence of a PRL-3/PTP1B/EGFR regulatory axis.
Despite the downregulation of PTP1B, which is also an SRCY530 phosphatase (50), PRL-3 was still able to decrease SRC-Y530 inhibitory phosphorylation and induce SRC activation. The regulation of SRC-Y530 phosphorylation is complex, with at least 2 kinases and 5 phosphatases known to regulate SRC-Y530 phosphorylation (51). In agreement with previous reports (52), we observed that PRL-3 overexpression in A431 cells resulted in the downregulation of CSK, an SRC-Y530-directed kinase (data not shown). Thus, it is likely that PRL-3 activates SRC via such alternative, non-PTP1B-dependent pathways. Although SRC was found to be a key regulator of EGFR activation (Figure 2, A and B), it was not the primary mechanism for PRL-3-induced EGFR byperactivation. Interestingly, besides activating the EGFR, SRC is also a downstream signal transducer from activated EGFR (53). In light of these observations, we propose that hyperactivation of EGFR by PRL-3 via PTP1B downregulation reflects an "umbrella effect," wherein activation of an upstream kinase (EGFR) promotes tumorigenesis by activation of multiple downstream oncogenic effectors - a phenomenon that could constitute a key node of PRL-3 oncogenic signaling (Figure 7). In agreement with this model, inhibition of SRC, AKT, or ERK1/2 kinases individually failed to generate any hypersensitive response in PRL-3-overexpressing cells (Supplemental Figure 4), likely due to the synergism between these pathways downstream of EGFR in regulating cellular growth and/or survival.

Most importantly, we present clinical evidence that high levels of PRL-3 expression induce sensitivity to EGFR inhibition. Indeed, high expression of PRL-3 in CRC patient tumors was a predictive marker of a favorable anti-EGFR therapeutic response to cetuximab monotherapy (Figure 6). Our result is consistent with a recent study by Baker et al. (40) investigating the expression signatures of 110 preselected genes in the cetuximab therapeutic response in CRC patients, where they identified elevated PRL-3 (PTP4A3) expression to be strongly associated with a favorable objective response, disease control, and progression-free survival. These independent results provide substantial support for our clinical findings that elevated PRL-3 expression is a predictive marker of a favorable anti-EGFR therapeutic response. Such dependence of PRL-3-expressing cells on EGFR signaling is reminiscent of the concept of "oncogene addiction" (37). The key implication of this concept is that terminating the signaling of a critical, hyperactivated pathway on which cancer cells have become dependent should have detrimental effects on cancer cell growth and survival, while sparing normal cells less reliant on these pathways. Many oncogenes have been described to confer such addiction (54). For instance, in non-small-cell lung carcinoma (NSCLC), activating mutations in EGFR or increased EGFR gene copy numbers have been found to both predict and underlie a better response to therapy using gefitinib, a clinical EGFR antagonist (55). Interestingly, we found higher expression of PRL-3 in gefitinib-sensitive compared with gefitinib-resistant NSCLC cell lines (Supplemental Figure 5), suggesting that PRL-3-induced addiction to EGFR might be a general property in multiple cancer types.

Targeting oncogene addiction has been proven to be a viable and effective approach in cancer therapy $(56,57)$. Here, we propose that PRL-3 hyperactivation of EGFR induces a therapeutically exploitable addiction to the receptor (Figure 7). Although further experimentation will be required to validate such dependence in different cancer models, our results suggest that targeting EGFR addiction presents an exciting and, given the availability of clinical anti- 
EGFR drugs (58), readily translatable therapeutic option in tumors overexpressing PRL-3. This is especially pertinent, given that no anti-PRL-3 agents have reached clinical trials, despite promising preclinical results $(59,60)$. In view of the observation that many carcinoma types, including lung, breast, and colon, display heightened EGFR activity, and given the clinical correlation of EGFR activity to tumor recurrence and shorter patient survival (11), the activation of EGFR by PRL-3 could be a key factor in cancer progression. Our results warrant further research on the validation of PRL-3 overexpression as a predictive biomarker for a favorable response to anti-EGFR therapy and propose EGFR inhibition as a viable and readily translatable therapeutic approach to effectively curtail the apparent synergy between $P R L-3$ and EGFR oncogenes.

\section{Methods}

Plasmids and siRNAs. The pEGFP-C1-PRL-3 construct has been described previously (61). Full-length PTP1B was PCR amplified from Addgene plasmid 8601/pJ3H-PTP1B (62) using primers containing a BamHI site (5'-CGGATCCATGGAGATGGAAAAGGAGTTC-3'; forward) or a NotI site (5'-TGCGGCCGCTATGTGTTGCTGTTGAACAGG-3'; reverse). PCR products were cloned into plasmid pXJ40-FLAG, a FLAG-tagged expression vector provided by Graeme Guy (Institute of Molecular and Cell Biology, Singapore) to generate pFLAG-PTP1B. All plasmids were verified by sequencing. Expression vectors encoding PRL-3-directed (5'-TTCTCGGCACCTTAAATTATT-3') or nontargeting scrambled shRNA sequences were commercially sourced (QIAGEN). siRNA against PTP1B (5'-CUUCCUAAGAACAAAAACC- $3^{\prime}$ ) were synthesized (1st Base) based on a previously validated sequence (63).

Cell culture and transfections. A2780 ovarian carcinoma cells and HCT116 colorectal carcinoma cells (ATCC) were routinely cultured in RPMI-1640 medium supplemented with $10 \%$ FBS, $2 \mathrm{mM}$ L-glutamine, and $1 \%$ antibiotic-antimycotic. A431 epidermoid carcinoma cells and MDA-MB-468 breast adenocarcinoma cells (ATCC) were grown in DMEM 4,500 mg/1 high-glucose medium supplemented with 10\% FBS, 2 mM L-glutamine, and $1 \%$ antibiotic-antimycotic. Cells were cultured in sterilized tissue culture-treated vessels at $37^{\circ} \mathrm{C}$ with $5 \% \mathrm{CO}_{2}$ and $95 \%$ humidity. Stable cell lines expressing EGFP (vec) or EGFP-PRL-3 (PRL-3) were generated by transfection of cells with pEGFP-C1 vector (Clontech) or PEGFP-C1-PRL-3 constructs using Lipofectamine 2000 (Invitrogen), followed by selection in 1 $\mathrm{mg} / \mathrm{ml}$ neomycin (Sigma-Aldrich) for 2 weeks to obtain pooled cell populations possessing greater than or equal to $90 \%$ GFP positivity under Eclipse TE2000-U fluorescence microscopy (Nikon). HCT116 cells stably expressing shRNA constructs were similarly generated, but were selected using 1 $\mu \mathrm{g} / \mathrm{ml}$ puromycin (Sigma-Aldrich) instead. For transient overexpression, cells were transfected with pFLAG-PTP1B or vector control plasmids for 18 hours using JetPrime (Polyplus Transfection) according to the manufacturer's protocol. Plasmid amounts were titrated ( $250 \mathrm{ng}$ ) to achieve FLAGPTP1B overexpression in MDA-MB-468-PRL-3 cells matching the endogenous PTP1B level in MDA-MB-468-vec cells as closely as possible (rescue experiment). For siRNA transfection, $10 \mathrm{nM}$ siRNA (final) was added to the cells previously seeded overnight in 12 -well plates using JetPrime reagent according to the manufacturer's protocol. Fresh medium was replaced 24 hours later, and the cells were incubated for another 48 hours before analysis. We found this siRNA transfection condition sufficient to deplete the expression of PTP1B in MDA-MB-468-vec cells to match the endogenous PTP1B level in MDA-MB-468-PRL-3 cells ("rescue" experiment).

Treatments. For EGF stimulation, cell monolayers (80\% confluent) were rinsed in PBS once before incubation in serum-free media for 16 hours. Recombinant EGF (100 ng/ml; Peprotech) was subsequently added 20 minutes prior to lysis. Unless otherwise specified, inhibitors were used at the following final concentrations: tryphostin AG-1478 (2 $\mu \mathrm{M}$; Calbiochem), cetuximab (10 nM; Zuellig Pharma), erlotinib (2 $\mu \mathrm{M}$; LC Labs), PP2 (5 $\mu \mathrm{M}$; Sigma-Aldrich), perifosine (1 $\mu \mathrm{M}$; LC Labs), U0126 (10 $\mu \mathrm{M}$; LC Labs), and cisplatin ( $1 \mu \mathrm{g} / \mathrm{ml}$; Pfizer). DMSO (0.1\% final; Merck) and PBS $(0.033 \%$ final) were used as negative controls for inhibitor treatments.

Western blot analysis. Western blotting was performed as described (64). Membranes were stained with antibodies against AKT, p-AKT S473, EGFR, p-EGFR Y845, p-EGFR Y1045, p-EGFR Y1068, p-ELK1 S383, ERK1/2, p-ERK1/2 T202/Y204, GAB1, GFP, GRB2, HER2, p-HER2 Y1221/2, PTP1B, SRC, p-SRC Y530, STAT5, p-STAT5 Y694 (all from Cell Signaling Technology), GAPDH, p-Tyr clone 4G10 (both from Millipore), ERK1, p-JNK T183/Y185, p-p38 T180/Y182, p-STAT1 Y701 (all from BD Biosciences), p-SHC Y239/240 (Santa Cruz Biotechnology Inc.), TC-PTP (R\&D Systems), or PRL-3 (64), followed by the appropriate secondary HRP-conjugated antibodies (Jackson ImmunoResearch Laboratories). The membranes were visualized using a chemiluminescent substrate (Thermo Scientific).

Antibody arrays. For the RTK activation study, antibody arrays against 71 unique tyrosine kinases (RayBio; catalog AAH-PRTK-1-2) were used according to the manufacturer's protocol. Cell lysates $(1.2 \mathrm{mg})$ were added to each membrane. Spot quantitation was done using a GS-700 Imaging Densitometer (Bio-Rad), with a fixed volume size for all spots being compared, and mean densities were calculated for each spot in duplicate (Supplemental Table 1; antibody map shown in Supplemental Figure 1). For the secreted growth factor analysis, $1 \mathrm{ml}$ of conditioned media from cells in DMEM without FBS (24-hour incubation) was added to antibody arrays against 41 unique growth factors (RayBio; catalog AAH-GF-1) and processed according to the manufacturer's protocol. The growth factor array antibody map is provided in Supplemental Figure 2.

$q R T-P C R$. RNA was extracted using an RNeasy Mini Kit (QIAGEN) according to the manufacturer's instructions, and $1 \mu \mathrm{g}$ was used for reverse transcription (Invitrogen). For qPCR, 100 ng of cDNA was amplified using a TaqMan Gene Expression Assay Mix with primers specific for human PTP1B/PTPN1 or GAPDH (Applied Biosystems). Real-time PCR was performed in triplicate wells using the 7500 Fast Real-Time PCR system (Applied Biosystems).

Immunofluorescence. Cells grown on coverglasses (Marienfeld-Superior) were treated with cetuximab $(100 \mathrm{nM})$ or erlotinib $(2 \mu \mathrm{M}) 16$ hours prior to fixation in $4 \%$ paraformaldehyde. Immunofluorescence microscopy was performed as previously described (64). For F-actin visualization, cells were stained with Alexa Fluor 568 phalloidin (Invitrogen) according to the manufacturer's protocol.

MTT (3-(4,5-dimethylthiazol-2-yl)-2,5-diphenyltetrazolium bromide) cell growth assay. Cells $\left(2 \times 10^{3}\right)$ were seeded in $10 \%$ FBS medium, placed in triplicate wells of a 96-well plate, and allowed to attach overnight. The medium was then replaced with $0.5 \%$ FBS medium in the presence or absence of various inhibitors and left to incubate for 48 hours. The medium was subsequently aspirated and replaced with $200 \mu \mathrm{l}$ of $0.5 \%$ FBS medium containing MTT $\left(0.2 \mathrm{mg} / \mathrm{ml}\right.$; Sigma-Aldrich) and left to incubate for 4 hours at $37^{\circ} \mathrm{C}$ at $5 \%$ $\mathrm{CO}_{2}$. Then, the supernatant was aspirated, $150 \mu \mathrm{l}$ of DMSO was added to each well, and absorbance was measured at $595 \mathrm{~nm}$. All experiments were done in triplicate and standard deviations were obtained. Results are expressed as the ratio of cell viability, calculated as: (absorbance of inhibitor-treated well minus absorbance of cell-free control)/(absorbance of control-treated well minus absorbance of cell-free control). Results were normalized to A431-vec DMSO-treated cells. No significant difference in cell proliferation was observed for A431-vec or A431-PRL-3 cells when treated with $0.1 \%$ DMSO (solvent for AG-1478 and erlotinib) or $0.033 \%$ PBS (solvent for cetuximab) (data not shown).

Monolayer clonogenicity assay. Three hundred cells were seeded in 10\% FBS medium, placed into $10-\mathrm{cm}$ dishes, and allowed to attach overnight. The 
medium was then replaced with $10 \%$ FBS medium with or without $20 \mathrm{nM}$ cetuximab and incubated for 10 days. To quantify colony formation, cells were washed twice with ice-cold PBS before fixing in ice-cold $20 \%$ methanol for 10 minutes. Subsequently, $0.5 \%$ crystal violet (w/v) in $25 \%$ methanol was used to stain colonies for 10 minutes and was thoroughly washed in deionized water before image acquisition. To score for actively dividing cells, colonies with diameters greater than or equal to $3 \mathrm{~mm}$ were counted and used in the statistical analysis.

Microarray dataset analysis. We analyzed 3 public gene expression profile (GEP) microarray datasets: the GSE12276 dataset consisting of 204 primary breast cancer specimens from patients with metastatic disease (31); the GSE5851 dataset consisting of 80 colorectal cancer specimens taken from patients prior to treatment with cetuximab (39); and the GSE4342 dataset consisting of a panel of NSCLC cell lines analyzed for sensitivity to and resistance against gefitinib treatment (41). All datasets were retrieved from the Gene Expression Omnibus (GEO) database and processed using $\mathrm{R}$ scripting as previously described (65). Since all 3 datasets were assayed on Affymetrix HG-U133 platforms, we used the average expression level for PRL-3/PTP4A3 probe sets ("206574_s_at" and "209695_at") or PTP1B/ PTPN1 probe sets ("202716_at", "217686_at", "217689_at" and "239526_x_ at") for further analysis. For the GSE12276 dataset, we investigated the association between $P R L-3$ and $P T P 1 B$ expression, as well as the correlation of $P R L-3$ with disease-free survival. Patients were divided into 2 groups ("high" or "low") based on their PRL-3 mRNA expression levels using the highest quartile value as a cutoff point. For the GSE5851 dataset, we analyzed the correlation between the tumors' baseline PRL-3 expression prior to cetuximab therapy and cetuximab response (as determined according to the Response Evaluation Criteria in Solid Tumors [RECIST]) as well as the resulting progression-free survival. We adopted the original study's 4 designations of cetuximab response (complete response, CR; partial response, PR; stable disease, SD; progressive disease, PD) to obtain 1 CR, 5 PR, 19 SD, and 43 PD samples (39). The responses for 12 patients could not be determined due to death before their first radiographic assessment, and these patients were thus excluded from our analysis. For the ORRs, patients were separated into CR/PR or SD/PD groups and compared. For DCRs, patients were separated into $\mathrm{CR} / \mathrm{PR} / \mathrm{SD}$ or PD groups and compared. For survival analysis, patients were divided into 2 groups ("high" or "low") based on their $P R L-3$ mRNA expression levels using their median values as a cutoff point. For the GSE4342 dataset, we analyzed the expression levels of PRL-3 in NSCLC cell lines (listed in Table 1 of the original publication; ref. 41) according to the references' designation of gefitinib-sensitive ( $\mathrm{IC}_{50} \leq 4.5$ $\mu \mathrm{mol} / 1, n=15)$ or gefitinib-resistant $\left(\mathrm{IC}_{50}>4.5 \mu \mathrm{mol} / 1, n=12\right)$.

Xenograft experiments. A431-vec or A431-PRL-3 cells $\left(3 \times 10^{6}\right)$ were injected s.c. into the left and right hips of 8-week-old nude mice (The
Jackson Laboratory) and allowed to grow until tumor volumes reached $30-40 \mathrm{~mm}^{3}$, after which the mice were divided into 2 groups and treated i.p. with $200 \mu \mathrm{l}$ of either PBS or cetuximab (1 mg) twice weekly for up to 14 days. Tumor size was measured twice weekly using calipers. Tumor volume in cubic millimeters was calculated using the formula $\pi / 6 \times$ (large diameter $) \times(\text { small diameter })^{2}$.

Statistics. For cell proliferation assays, the Student's $t$ test was used to test for significant differences. For qRT-PCR, the paired Student's $t$ test was used to analyze biological triplicates for significance. For xenograft tumors, growth curves were compared using 2-way ANOVA, whereas the differences in final tumor volumes between groups were compared using the Student's $t$ test. In analyzing the human breast cancer sample microarray dataset, the association between raw $P R L-3$ and $P T P 1 B$ mRNA expression was analyzed using Spearman's rank test. Further correlation between stratified $P R L-3$ expression and PTP1B expression was tested using the Mann-Whitney $U$ test. The correlation between $P R L-3$ expression and cetuximab treatment responses (objective response and disease control) was tested using the Student's $t$ test and was further complemented by Fisher's exact test for treatment response rates (ORR, DCR). Similarly, the correlation between $P R L-3$ expression and gefitinib sensitivity/resistance was tested using the Student's $t$ test. Kaplan-Meier analysis, in conjunction with Cox proportional hazard regression analysis, were used to test the correlation between $P R L-3$ expression groups (high or low) and patient survival. Statistical analysis was performed using the SPSS 15.0 software package (IBM), and $P$ values less than 0.05 were considered statistically significant.

Study approval. All animal studies were approved by the IACUC of the Biological Resource Centre, A*STAR, Singapore.

\section{Acknowledgments}

We are very grateful to John Wong and Patrick Tan for their assistance in sourcing clinical data. We thank Hong Wanjin for his critical reading of the manuscript, and Graeme Guy and Ben Neel for providing pXJ40-FLAG and PJ3H-PTP1B plasmids, respectively. Finally, we thank Thura Min and Li Jie for their technical assistance with xenograft experiments. This work was supported by research grants from the Agency of Science, Technology and Research (A*STAR), Singapore.

Received for publication September 12, 2012, and accepted in revised form May 10, 2013.

Address correspondence to: Qi Zeng, 61 Biopolis Drive, Proteos, Room 3-02B, Singapore 138673. Phone: 65.6586.9664; Fax: 65.6779.1117; E-mail: mcbzengq@imcb.a-star.edu.sg.
1. Tonks NK. Protein tyrosine phosphatases: from genes, to function, to disease. Nat Rev Mol Cell Biol. 2006;7(11):833-846.

2. Saha $\mathrm{S}$, et al. A phosphatase associated with metastasis of colorectal cancer. Science. 2001; 294(5545):1343-1346.

3. Laurent C, et al. High PTP4A3 phosphatase expression correlates with metastatic risk in uveal melanoma patients. Cancer Res. 2011;71(3):666-674

4. Bessette DC, Wong PCW, Pallen CJ. PRL-3: a metastasis-associated phosphatase in search of a function. Cells Tissues Organs. 2007;185(1-3):232-236.

5. Al-Aidaroos AQ, Zeng Q. PRL-3 phosphatase and cancer metastasis. J Cell Biochem. 2010. 111(5):1087-1098.

6. Wang H, Quah SY, Dong JM, Manser E, Tang JP, Zeng Q. PRL-3 down-regulates PTEN expression and signals through PI3K to promote epithelial-mesenchymal transition. Cancer Res. 2007;67(7):2922-2926.
7. Wang $\mathrm{H}$, et al. PCBP1 suppresses the translation of metastasis-associated PRL-3 phosphatase. Cancer Cell. 2010;18(1):52-62.

8. Wee S, et al. PTEN-deficient cancers depend on PIK3CB. Proc Natl Acad Sci U S A. 2008; 105(35):13057-13062.

9. Yarden Y, Sliwkowski MX. Untangling the ErbB signalling network. Nat Rev Mol Cell Biol. 2001;2(2):127-137.

10. Olayioye MA, Neve RM, Lane HA, Hynes NE. The ErbB signaling network: receptor heterodimerization in development and cancer. $E M B O J$. 2000;19(13):3159-3167.

11. Yarden Y, Pines G. The ERBB network: at last, cancer therapy meets systems biology. Nat Rev Cancer. 2012;12(8):553-563.

12. Sridhar SS, Seymour L, Shepherd FA. Inhibitors of epidermal-growth-factor receptors: a review of clinical research with a focus on non-small-cell lung cancer. Lancet Oncol. 2003;4(7):397-406.
13. Yakes FM, Chinratanalab W, Ritter CA, King W, Seelig S, Arteaga CL. Herceptin-induced inhibition of phosphatidylinositol-3 kinase and Akt Is required for antibody-mediated effects on p27, cyclin D1, and antitumor action. Cancer Res. 2002;62(14):4132-4141.

14. Ebi H, et al. Receptor tyrosine kinases exert dominant control over PI3K signaling in human KRAS mutant colorectal cancers. J Clin Invest. 2011;121(11):4311-4321.

15. Merlino GT, et al. Amplification and enhanced expression of the epidermal growth factor receptor gene in A431 human carcinoma cells. Science. 1984;224(4647):417-4119.

16. Ciaccio MF, Wagner JP, Chuu CP, Lauffenburger DA, Jones RB. Systems analysis of EGF receptor signaling dynamics with microwestern arrays. Nat Methods. 2010;7(2):148-155.

17. Astsaturov I, et al. Synthetic lethal screen of an EGFR-centered network to improve targeted ther- 
apies. Sci Signal. 2010;3(140):ra67.

18. Rojas M, Yao S, Lin YZ. Controlling epidermal growth factor (EGF)-stimulated Ras activation in intact cells by a cell-permeable peptide mimicking phosphorylated EGF receptor. J Biol Chem. 1996;271(44):27456-27461.

19. Batzer AG, Rotin D, Ureña JM, Skolnik EY, Schlessinger J. Hierarchy of binding sites for Grb2 and She on the epidermal growth factor receptor. Mol Cell Biol. 1994;14(8):5192-5201.

20. Rodrigues GA, Falasca M, Zhang Z, Ong SH, Schlessinger J. A novel positive feedback loop mediated by the docking protein Gab1 and phosphatidylinositol 3-kinase in epidermal growth factor receptor signaling. Mol Cell Biol. 2000;20(4):1448-1459.

21. Liang F, Liang J, Wang WQ, Sun JP, Udho E, Zhang ZY. PRL3 promotes cell invasion and proliferation by down-regulation of Csk leading to Src activation. J Biol Chem. 2007;282(8):5413-5419.

22. Bromann PA, Korkaya H, Courtneidge SA. The interplay between Src family kinases and receptor tyrosine kinases. Oncogene. 2004;23(48):7957-7968.

23. Tice DA, Biscardi JS, Nickles AL, Parsons SJ. Mechanism of biological synergy between cellular Src and epidermal growth factor receptor. Proc Natl Acad Sci U S A. 1999;96(4):1415-1420.

24. Levitzki A, Gazit A. Tyrosine kinase inhibition: an approach to drug development. Science. 1995;267(5205):1782-1788

25. Okutani Y, et al. Src directly tyrosine-phosphorylates STAT5 on its activation site and is involved in erythropoietin-induced signaling pathway. Oncogene. 2001;20(45):6643-6650.

26. Ceresa BP, Vanlandingham PA. Molecular mechanisms that regulate epidermal growth factor receptor inactivation. Clin Med Oncol. 2008;2:47-61.

27. Haj FG, Markova B, Klaman LD, Bohmer FD, Neel BG. Regulation of receptor tyrosine kinase signaling by protein tyrosine phosphatase-1B. J Biol Chem. 2003;278(2):739-744.

28. Sangwan V, et al. Protein tyrosine phosphatase PTP1B modulates early endosome fusion and trafficking of Met and EGF receptor tyrosine kinases. J Biol Chem. 2011;286(52):45000-45013.

29. Gridelli C, Bareschino MA, Schettino C, Rossi A, Maione P, Ciardiello F. Erlotinib in non-small cell lung cancer treatment: current status and future development. Oncologist. 2007;12(7):840-849.

30. Vincenzi B, Schiavon G, Silletta M, Santini D, Tonini G. The biological properties of cetuximab. Crit Rev Oncol Hematol. 2008;68(2):93-106.

31. Bos PD, et al. Genes that mediate breast cancer metastasis to the brain. Nature. 2009; 459(7249):1005-1009.

32. Wehrle-Haller B, Imhof BA. Actin, microtubules and focal adhesion dynamics during cell migration. Int J Biochem Cell Biol. 2003;35(1):39-50.

33. Thiery JP, Sleeman JP. Complex networks orchestrate epithelial-mesenchymal transitions. Nat Rev
Mol Cell Biol. 2006;7(2):131-142.

34. Yip SC, et al. The distinct roles of Ras and Rac in PI 3-kinase-dependent protrusion during EGF-stimulated cell migration. J Cell Sci. 2007; 120(pt 17):3138-3146.

35. Bianco R, et al. Loss of PTEN/MMAC1/TEP in EGF receptor-expressing tumor cells counteracts the antitumor action of EGFR tyrosine kinase inhibitors. Oncogene. 2003;22(18):2812-2822.

36. Yamasaki F, et al. Sensitivity of breast cancer cells to erlotinib depends on cyclin-dependent kinase 2 activity. Mol Cancer Ther. 2007;6(8):2168-2177.

37. Weinstein IB, Joe A. Oncogene addiction. Cancer Res. 2008;68(9):3077-3080.

38. [No authors listed]. Platinum complexes in cancer chemotherapy. Antibiot Chemother. 1978;23:99-112.

39. Khambata-Ford S, et al. Expression of epiregulin and amphiregulin and K-ras mutation status predict disease control in metastatic colorectal cancer patients treated with cetuximab. J Clin Oncol. 2007;25(22):3230-3237.

40. Baker JB, et al. Tumour gene expression predicts response to cetuximab in patients with KRAS wild-type metastatic colorectal cancer. Br J Cancer. 2011;104(3):488-495

41. Coldren $C D$, et al. Baseline gene expression predicts sensitivity to gefitinib in non-small cell lung cancer cell lines. Mol Cancer Res. 2006;4(8):521-528.

42. Robertson SC, Tynan J, Donoghue DJ. RTK mutations and human syndromes: when good receptors turn bad. Trends Genet. 2000;16(8):368.

43. Bennett H, et al. Signalling by EGF receptor in human cancers: Accentuate the positive, eliminate the negative. In: Haley J, Gullick W, eds. EGFR Signaling Networks in Cancer Therapy. New York, New York, USA: Humana Press; 2009:224-244.

44. Schlessinger J. Cell signaling by receptor tyrosine kinases. Cell. 2000;103(2):211-225.

45. Ferrari E, et al. Identification of new substrates of the protein-tyrosine phosphatase PTP1B by Bayesian integration of proteome evidence.J Biol Chem. 2011;286(6):4173-4185

46. Fukada T, Tonks NK. Identification of YB-1 as a regulator of PTP1B expression: implications for regulation of insulin and cytokine signaling. EMBO J. 2003;22(3):479-493.

47. Suwaki N, et al. A HIF-regulated VHL-PTP$1 \mathrm{~B}-\mathrm{Src}$ signaling axis identifies a therapeutic target in renal cell carcinoma. Sci Transl Med. 2011;3(85):85ra47.

48. Fukada T, Tonks NK. The reciprocal role of Egr-1 and Sp family proteins in regulation of the PTP1B promoter in response to the $\mathrm{p} 210$ $\mathrm{Bcr}-\mathrm{Abl}$ oncoprotein-tyrosine kinase. J Biol Chem. 2001;276(27):25512-25519.

49. Liu Y, et al. An epigenetic role for PRL-3 as a regulator of $\mathrm{H} 3 \mathrm{~K} 9$ methylation in colorectal cancer. Gut. 2013;62(4):571-581.

50. Zhu S, Bjorge JD, Fujita DJ. PTP1B contrib- utes to the oncogenic properties of colon cancer cells through Src activation. Cancer Res. 2007;67(21):10129-10137.

51. Sen B, Johnson FM. Regulation of SRC family kinases in human cancers. J Signal Transduct. 2011;2011:865819.

52. Liang F, et al. Translational control of C-terminal Src kinase (Csk) expression by PRL3 phosphatase. J Biol Chem. 2008;283(16):10339-10346.

53. Jorissen RN, Walker F, Pouliot N, Garrett TPJ, Ward CW, Burgess AW. Epidermal growth factor receptor: mechanisms of activation and signalling. In: Carpenter G, ed. The EGF Receptor Family. Nashville, Tennessee, USA: Academic Press; 2003:33-55.

54. Sharma SV, Settleman J. Oncogene addiction: setting the stage for molecularly targeted cancer therapy. Genes Dev. 2007;21(24):3214-3231.

55. Takano T, et al. Epidermal growth factor receptor gene mutations and increased copy numbers predict gefitinib sensitivity in patients with recurrent non-small-cell lung cancer. J Clin Oncol. 2005;23(28):6829-6837.

56. Druker BJ, et al. Efficacy and safety of a specific inhibitor of the BCR-ABL tyrosine kinase in chronic myeloid leukemia. $N$ Engl J Med. 2001;344(14):1031-1037.

57. Zuber J, et al. An integrated approach to dissecting oncogene addiction implicates a Myb-coordinated self-renewal program as essential for leukemia maintenance. Genes Dev. 2011;25(15):1628-1640.

58. Mortlock AA, Barker AJ. Kinase inhibitors for cancer. In: Taylor JB, Triggle DJ, eds. Comprehensive Medicinal Chemistry II. London, United Kingdom: Elsevier Science Ltd.; 2007:183-220.

59. Daouti S, et al. A selective phosphatase of regenerating liver phosphatase inhibitor suppresses tumor cell anchorage-independent growth by a novel mechanism involving $\mathrm{p} 130 \mathrm{Cas}$ cleavage. Cancer Res. 2008;68(4):1162-1169.

60. Guo K, et al. Engineering the first chimeric antibody in targeting intracellular PRL-3 oncoprotein for cancer therapy in mice. Oncotarget. 2012;3(2):158-171.

61. Guo K, et al. PRL-3 initiates tumor angiogenesis by recruiting endothelial cells in vitro and in vivo. Cancer Res. 2006;66(19):9625-9635.

62. Sells MA, Chernoff J. Epitope-tag vectors for eukaryotic protein production. Gene. 1995;152(2):187-189.

63. Arias-Romero LE, et al. Activation of Src by protein tyrosine phosphatase $1 \mathrm{~B}$ Is required for ErbB2 transformation of human breast epithelial cells. Cancer Res. 2009;69(11):4582-4588.

64. Li J, et al. Generation of PRL-3- and PRL-1-specific monoclonal antibodies as potential diagnostic markers for cancer metastases. Clin Cancer Res. 2005;11(6):2195-2204.

65. Yuen HF, et al. RanGTPase: a candidate for Mycmediated cancer progression. J Natl Cancer Inst. 2013;105(7):475-488. 